\title{
1-d Quantum Harmonic Oscillator with Time Quasi-periodic Quadratic Perturbation: Reducibility and Growth of Sobolev Norms
}

\author{
Zhenguo Liang ${ }^{\text {a }}{ }^{1}$, Zhiyan Zhao ${ }^{\mathrm{b}, 2}$, Qi Zhou ${ }^{\mathrm{c}, 3}$ \\ a School of Mathematical Sciences and Key Lab of Mathematics for Nonlinear Science, \\ Fudan University, Shanghai 200433, China \\ b Université Côte d'Azur, CNRS, Laboratoire J. A. Dieudonné, 06108 Nice, France \\ ${ }^{c}$ Chern Institute of Mathematics and LPMC, Nankai University, Tianjin 300071, China
}

\begin{abstract}
For a family of 1-d quantum harmonic oscillators with a perturbation which is $C^{2}$ parametrized by $E \in \mathcal{I} \subset \mathbb{R}$ and quadratic on $x$ and $-\mathrm{i} \partial_{x}$ with coefficients quasi-periodically depending on time $t$, we show the reducibility (i.e., conjugation to time-independent) for a.e. $E$. As an application of reducibility, we describe the behaviors of solutions in Sobolev space:

- Boundedness w.r.t. $t$ is always true for "most" $E \in \mathcal{I}$.

- For "generic" time-dependent perturbation, polynomial growth and exponential growth to infinity w.r.t. $t$ occur for $E$ in a "small" part of $\mathcal{I}$.
\end{abstract}

\footnotetext{
${ }^{1}$ Z. Liang was partially supported by NSFC grant $(11371097,11571249)$ and Natural Science Foundation of Shanghai (19ZR1402400).

${ }^{2}$ Corresponding author. The work of Z. Zhao was partially supported by the French government through the National Research Agency (ANR) grant ANR-15-CE40-0001-03 for the project BeKAM and through the UCA JEDI Investments in the Future project managed by ANR with the reference number ANR-15-IDEX-01

${ }^{3}$ Q. Zhou was partially supported by support by NSFC grant (11671192, 11771077), the Science Fund for Distinguished Young Scholars of Tianjin (No. 19JCJQJC61300) and Nankai Zhide Foundation.

E-mail addresses: zgliang@fudan.edu.cn(Z. Liang), zhiyan.zhao@univ-cotedazur.fr(Z. Zhao), qizhou@nankai.edu.cn(Q. Zhou)
} 
Concrete examples are given for which the growths of Sobolev norm do occur.

\section{Résumé}

Pour une famille des oscillateurs harmoniques quantiques unidimensionnels avec une perturbation qui est parametrée par $E \in \mathcal{I} \subset \mathbb{R}$ d'une manière $C^{2}$ et qui est quadratique sur $x$ et $-\mathrm{i} \partial_{x}$ avec des coefficients qui dépendent du temps $t$ d'une manière quasi-periodique, on montre la réductibilité (c'est-àdire la conjugaison à l'indépendant du temps) pour presque tout $E$. Comme une application de la réductibilité, on décrit les comportements des solutions dans l'espace de Sobolev:

- La bornitude par rapport à $t$ est toujours vraie pour la « plupart $\gg$ de $E \in \mathcal{I}$.

- Pour la perturbation « générique 》qui dépend du temps, la croissance polynomiale et la croissance exponentielle à l'infini par rapport à $t$ ont lieu pour $E$ dans une $\ll$ petite $\gg$ partie de $\mathcal{I}$.

Des exemples concrets sont donnés pour lesquels les croissances de la norme de Sobolev vraiment ont lieu.

Keywords: 1-d quantum harmonic oscillator; time quasi-periodic; reducibility; growth of Sobolev norms 2010 MSC: 35Q40; 35Q41; 47G30

\section{Introduction and main results}

Consider the one-dimensional Schrödinger equation

$$
\mathrm{i} \partial_{t} u=\frac{\nu(E)}{2} H_{0} u+W\left(E, \omega t, x,-\mathrm{i} \partial_{x}\right) u, \quad x \in \mathbb{R},
$$

where, we assume that

- the frequencies $\omega \in \mathbb{R}^{d}, d \geq 1$, satisfy the Diophantine condition (denoted by $\omega \in \mathrm{DC}_{d}(\gamma, \tau)$ for $\left.\gamma>0, \tau>d-1\right)$ :

$$
\inf _{j \in \mathbb{Z}}|\langle n, \omega\rangle-j|>\frac{\gamma}{|n|^{\tau}}, \quad \forall n \in \mathbb{Z}^{d} \backslash\{0\},
$$

- the parameter $E \in \mathcal{I}$, an interval $\subset \mathbb{R}$, and $\nu \in C^{2}(\mathcal{I}, \mathbb{R})$ satisfies

$$
\left|\nu^{\prime}(E)\right| \geq l_{1}, \quad\left|\nu^{\prime \prime}(E)\right| \leq l_{2}, \quad \forall E \in \mathcal{I},
$$

for some $l_{1}, l_{2}>0$, 
- $H_{0}$ is the one-dimensional quantum harmonic oscillator, i.e.

$$
\left(H_{0} u\right)(x):=-\left(\partial_{x}^{2} u\right)(x)+x^{2} \cdot u(x), \quad \forall u \in L^{2}(\mathbb{R}),
$$

- $W(E, \theta, x, \xi)$ is a quadratic form of $(x, \xi)$ :

$$
W(E, \theta, x, \xi)=\frac{1}{2}\left(a(E, \theta) x^{2}+2 b(E, \theta) x \cdot \xi+c(E, \theta) \xi^{2}\right),
$$

with $a, b, c: \mathcal{I} \times \mathbb{T}^{d} \rightarrow \mathbb{R}$, all of which are $C^{2}$ w.r.t. $E \in \mathcal{I}$ and $C^{\omega}$ w.r.t. $\theta \in \mathbb{T}^{d}:=(\mathbb{R} / \mathbb{Z})^{d}$, and for every $E \in \mathcal{I}$, for $m=0,1,2$, $\left|\partial_{E}^{m} a(E, \cdot)\right|_{r}:=\sup _{|\Im z|<r}\left|\partial_{E}^{m} a(E, z)\right|,\left|\partial_{E}^{m} b(E, \cdot)\right|_{r},\left|\partial_{E}^{m} c(E, \cdot)\right|_{r}$ are small enough.

We will prove that, for almost every $E$ in the interval $\mathcal{I}$, Eq. (1) is reducible, i.e., via a unitary transformation, Eq. (11) is conjugated to an equation which is independent of time (while the transformation depends on time in an analytic quasi-periodic way). According to the reducibility, we deduce the behavior of Sobolev norms for the solutions to Eq. (11).

\subsection{Reducibility for harmonic oscillators}

Our main result is the following:

Theorem 1. There exists $\varepsilon_{*}=\varepsilon_{*}\left(\gamma, \tau, r, d, l_{1}, l_{2}\right)>0$ such that if

$$
\max _{m=0,1,2}\left\{\left|\partial_{E}^{m} a\right|_{r},\left|\partial_{E}^{m} b\right|_{r},\left|\partial_{E}^{m} c\right|_{r}\right\}=: \varepsilon_{0} \leq \varepsilon_{*}, \quad \forall E \in \mathcal{I},
$$

then for a.e. $E \in \mathcal{I}, E q$. (1) is reducible, i.e., there exists a time quasiperiodic transformation $U(\omega t)$, unitary in $L^{2}$ and analytically depending on $t$, such that Eq. (1) is conjugated to $\mathrm{i}_{t} v=G v$ by the transformation $u=$ $U(\omega t) v$, with $G$ a linear operator independent of $t$.

More precisely, there exists a subset

$$
\mathcal{O}_{\varepsilon_{0}}=\bigcup_{j \in \mathbb{N}} \Lambda_{j} \subset \overline{\mathcal{I}}
$$

with $\Lambda_{j}$ 's being closed intervals $\frac{1}{4}$ and $\operatorname{Leb}\left(\mathcal{O}_{\varepsilon_{0}}\right)<\varepsilon_{0}^{\frac{1}{40}}$, such that the following holds.

\footnotetext{
${ }^{4}$ In this paper, the "closed interval" is interpreted in a more general sense, i.e., it can be degenerated to a point instead of a positive-measure subset of $\mathbb{R}$.
} 
1. For a.e. $E \in \mathcal{I} \backslash \mathcal{O}_{\varepsilon_{0}}, G$ is unitary equivalent to $\varrho H_{0}$ for some $\varrho=$ $\varrho_{E} \geq 0$

2. If $\operatorname{Leb}\left(\Lambda_{j}\right)>0$, then

- for $E \in \operatorname{int} \Lambda_{j}, G$ is unitary equivalent to $-\frac{\lambda i}{2}\left(x \cdot \partial_{x}+\partial_{x} \cdot x\right)$ for some $\lambda=\lambda_{E}>0$;

- for $E \in \partial \Lambda_{j} \backslash \partial \mathcal{I}, G$ is unitary equivalent to $-\frac{\kappa}{2} x^{2}$ for some $\kappa=$ $\kappa_{E} \in \mathbb{R} \backslash\{0\}$.

If $\operatorname{Leb}\left(\Lambda_{j}\right)=0$, then $G=0$ for $E \in \Lambda_{j}$.

Before giving its application on the growth of Sobolev norm, let us first make a review on previous works about the reducibility on harmonic oscillators as well as the relative KAM theory.

For 1-d harmonic oscillators with time periodic smooth perturbations, Combescure 11] firstly showed the pure point nature of Floquet operator (see also [13, 17, 29]). For 1-d harmonic oscillators with time quasi-periodic bounded perturbations, we can refer to [23, 39, 40] for the reducibility and the pure point spectrum of Floquet operator. For 1-d harmonic oscillators with unbounded time quasi-periodic perturbations, similar results can be found in [3, 4, 9, 31]. In investigating the reducibility problems, KAM theory for 1-d PDEs has been well developed by Bambusi-Graffi [6] and Liu-Yuan [33] in order to deal with unbounded perturbations.

Reducibility for PDEs in higher-dimensional case was initiated by EliassonKuksin [15], based on their KAM theory [16]. We refer to [24] and [32] for higher-dimensional harmonic oscillator with bounded potential. We mention that some higher-dimensional results with unbounded perturbations have been recently obtained [5, 19, 20, 21, 36]. However, a general KAM theorem for higher-dimensional PDEs with unbounded perturbations is far from success.

Recently, Bambusi-Grébert-Maspero-Robert [7] built a reducibility result for the harmonic oscillators on $\mathbb{R}^{n},{ }_{,} \geq 1$, in which the perturbation is a polynomial of degree at most two in $x$ and $-\mathrm{i} \partial_{x}$ with coefficients quasiperiodically depending on time. The proof in [7] exploits the fact that for polynomial Hamiltonians of degree at most 2, there is an exact correspondence between classical and quantum mechanics, so that the result can be proved by exact quantization of the classical KAM theory which ensures reducibility of the classical Hamiltonian system. The exact correspondence 
between classical and quantum dynamics of quadratic Hamiltonians was already exploited in the paper [26] to prove stability and instability results for one degree of freedom time periodic quadratic Hamiltonians. To prove our main result, we use the same strategy as [7] and the reducibility result for the classical Hamiltonian by Eliasson [14].

\subsection{Growth of Sobolev norms}

Besides reducibility, the construction of unbounded solutions in Sobolev space for Schrödinger equations attracts even more attentions.

As an application of Theorem 1, we can study the long time behaviour of its solution $u(t)$ to Eq. (11) in Sobolev space. For $s \geq 0$, we define Sobolev space

$$
\mathcal{H}^{s}:=\left\{\psi \in L^{2}(\mathbb{R}): H_{0}^{\frac{s}{2}} \psi \in L^{2}(\mathbb{R})\right\}
$$

and Sobolev norm $\|\psi\|_{s}:=\left\|H_{0}^{\frac{s}{2}} \psi\right\|_{L^{2}(\mathbb{R})}$. It is well known that, for $s \in \mathbb{N}$, the above definition of norm is equivalent to

$$
\sum_{\substack{\alpha+\beta \leq s \\ \alpha, \beta \in \mathbb{N}}}\left\|x^{\alpha} \cdot \partial^{\beta} \psi\right\|_{L^{2}(\mathbb{R})} .
$$

Remark 1.1. In view of Remark 2.2 of [9], we get that, for a given $\psi \in \mathcal{H}^{s}$,

$$
\|\psi\|_{s} \simeq\|\psi\|_{H^{s}}+\left\|x^{s} \psi\right\|_{L^{2}}
$$

replacing $K_{0}=H_{0}$ in that remark by $K_{0}=H_{0}^{\frac{1}{2}}$, where $H^{s}$ means the standard Sobolev space and $\|\cdot\|_{H^{s}}$ is the corresponding norm. Hence, to calculate the norm $\|\psi\|_{s}, s \geq 0$, it is sufficient to focus on $\left\|x^{s} \psi\right\|_{L^{2}}$ for $s \geq 0$ and $\left\|\psi^{(s)}\right\|_{L^{2}}$ for $s \in \mathbb{N}$.

For different types of reduced systems, Sobolev norm of solution exhibits different behaviors.

Theorem 2. Under the assumption of Theorem 1, for any $s \geq 0$, and any non-vanishing initial condition $u(0) \in \mathcal{H}^{s}$, the following holds true for the solution $u(t)$ to $E q$. (1) for $t \geq 0$.

1. For a.e. $E \in \mathcal{I} \backslash \mathcal{O}_{\varepsilon_{0}}, c \leq\|u(t)\|_{s} \leq C$.

2. If $\operatorname{Leb}\left(\Lambda_{j}\right)>0$, then 
- for $E \in \operatorname{int} \Lambda_{j}, c e^{\lambda s t} \leq\|u(t)\|_{s} \leq C e^{\lambda s t}$,

- for $E \in \partial \Lambda_{j} \backslash \partial \mathcal{I}, c|\kappa|^{s} t^{s} \leq\|u(t)\|_{s} \leq C|\kappa|^{s}\left(1+t^{2}\right)^{\frac{s}{2}}$.

If $\operatorname{Leb}\left(\Lambda_{j}\right)=0$, then for $E \in \Lambda_{j}, c \leq\|u(t)\|_{s} \leq C$.

Here $\lambda=\lambda_{E}$ and $\kappa=\kappa_{E}$ are the same with Theorem 1 and $c, C>0$ are two constants depending on $s, E$ and $u(0)$.

Remark 1.2. For a.e. $E \in \mathcal{I} \backslash \mathcal{O}_{\varepsilon_{0}}$, we have $0<c<C<\infty$. However, we could not expect the uniformity of these two constants and the ratio between them w.r.t. E. Indeed, the two constants are indeed influenced by the quasiperiodic unitary transformation $U(\omega t)$ and the (constant) unitary equivalence of $G$ obtained in Theorem [1. Since both unitary transformations may not be close to identity, $c$ and $C$ are usually not close to each other. Even though in the "simplest" case, i.e., no resonance occurs in the classical and quantum KAM iteration (see Proof of Proposition 5 (1) for details) and hence U( $\omega t)$ is close to identity, the ratio between $c$ and $C$ is not always close to 1 because of the nondeterminacy of $G$ (see Remark 1.2 of [M]). Moreover, different $E$ would have different scales of resonances, then $c, C$ would lose uniform control w.r.t E.

Let us review the progress on constructing unbounded solutions of timedependent Schrödinger equations. For linear Schrödinger equation on $\mathbb{T}$ with time quasi-periodic perturbation, by exploiting resonance effects, Bourgain [10] built logarithmic upper bound for Sobolev norm of solutions and constructed examples of solution which exhibits logarithmic growth with $t$. Later, for 1-d harmonic oscillator with certain time periodic order zero perturbation, Delort [12] constructed some solution with its Sobolev norm polynomially growing with $t$. By exploiting the idea in [22], Maspero [35] gave a simplified proof for the result of Delort [12]. In [7], the authors also considered the higher-dimensional harmonic oscillator with time quasi-periodic perturbation which is linear in $x$ and $-\mathrm{i} \partial_{x}$. Under the Diophantine condition on frequencies, the time-dependent equation can be reduced to a special "normal form" independent of time (see Theorem 3.3 of [7]), which implies the polynomial growth of Sobolev norm. In particular, a concrete example of such polynomial growth is given for 1-d harmonic oscillator with time periodic perturbation (see Corollary A.2 of [7]). Recently, for 2-d harmonic oscillator with perturbation which is decaying in $t$, Faou-Raphaël [18] constructed a solution whose $\mathcal{H}^{1}$-norm presents logarithmic growth with $t$. For 2-d harmonic oscillator with perturbation being the projection onto Bargmann-Fock 
space, Thomann 38] constructed explicitly a travelling wave whose Sobolev norm presents polynomial growth with $t$, based on the study in [37] for linear Lowest Landau Level equations (LLL) with a time-dependent potential. There are also many literatures, e.g., [8, 34], which are relative to the upper growth bound of the solution in Sobolev space.

From the above mentioned literatures, we can see that the growth of Sobolev norm of solution is closely related to the resonance phenomenon. However, it was not clear to us how growth and boundedness coexist with each other. Following [14], for 1-d harmonic oscillator with quadratic perturbation (11), we introduce in this paper the parameter set $\bigcup_{j \in \mathbb{N}} \Lambda_{j}$, in which the solutions have exponential growth with $t$, while on the boundaries of this set the solutions present polynomial growth with $t$. This subset gives a geometric description on the transition between boundedness and two types of growth.

\subsection{Examples with $\operatorname{Leb}\left(\mathcal{O}_{\varepsilon_{0}}\right)>0$}

In the following, we will present several concrete examples of time quasiperiodic quadratic perturbations for which the set $\bigcup_{j \in \mathbb{N}} \Lambda_{j}$ is of positive measure.

In view of Theorem 1 and 2, the growth of Sobolev norm can be obtained via the reducibility if $\operatorname{Leb}\left(\mathcal{O}_{\varepsilon_{0}}\right)>0$. We need to point that the time-dependent quadratic perturbation $W\left(E, \omega t, x,-\mathrm{i} \partial_{x}\right)$ with $\operatorname{Leb}\left(\mathcal{O}_{\varepsilon_{0}}\right)>0$ exists universally. In other words, it is a quite "extreme" case that

$$
\operatorname{Leb}\left(\Lambda_{j}\right)=0, \quad \forall j \in \mathbb{N} .
$$

We have the following concrete examples.

For $\mathcal{I}=\mathbb{R}, \nu(E)=E$, the equation

$$
\mathrm{i} \partial_{t} u=\frac{E}{2} H_{0} u+\left(\frac{a(\omega t)}{2} x^{2}-\frac{b(\omega t)}{2}\left(x \cdot \mathrm{i} \partial_{x}+\mathrm{i} \partial_{x} \cdot x\right)-\frac{c(\omega t)}{2} \partial_{x}^{2}\right) u,
$$

satisfies the assumptions of Theorem 1 if $a, b, c \in C^{\omega}\left(\mathbb{T}^{d}, \mathbb{R}\right)$ are small enough. Hence, for Eq. (3), the reducibility and the behaviors of $\mathcal{H}^{s}$ norm of solutions described in Theorem 2 can be obtained.

Theorem 3. For generic $a, b, c \in C^{\omega}\left(\mathbb{T}^{d}, \mathbb{R}\right)$ with $|a|_{r},|b|_{r},|c|_{r}$ small enough (depending on $r, \gamma, \tau, d)$, the conclusions of Theorem 1 and 2 hold for Eq. (3) for $\mathcal{I}=\mathbb{R}$ with $\operatorname{Leb}\left(\mathcal{O}_{\varepsilon_{0}}\right)>0$. 
For $\nu(E)=\sqrt{E}$, consider the equation

$$
\mathrm{i} \partial_{t} u=\frac{\sqrt{E}}{2} H_{0} u-\frac{q(\omega t)}{2 \sqrt{E}}\left(x^{2}-x \cdot \mathrm{i} \partial_{x}-\mathrm{i} \partial_{x} \cdot x-\partial_{x}^{2}\right) u .
$$

with $q \in C_{r}^{\omega}\left(\mathbb{T}^{d}, \mathbb{R}\right)$. The equation is important, since as we will show later, it is closely related to quasi-periodic Schrödinger operator.

Theorem 4. For generic $q \in C^{\omega}\left(\mathbb{T}^{d}, \mathbb{R}\right)$, the conclusions of Theorem 1 and 2 hold for $E q$. (4) for $\mathcal{I}=\left[E_{0}, E_{1}\right]$ with $\operatorname{Leb}\left(\Lambda_{j}\right)>0$ for infinitely many $j$ 's, where $E_{0}>0$ is large enough (depending on $|q|_{r}$ ) and $E_{1}<\infty$.

Theorem 3 gives the example that $\operatorname{Leb}\left(\Lambda_{j}\right)>0$ for at least one $j$, while Theorem 4 gives the example that $\operatorname{Leb}\left(\Lambda_{j}\right)>0$ for infinitely many $j$ 's. Indeed, if the dimension of the frequency $d=2$, we could even give examples for which $\operatorname{Leb}\left(\Lambda_{j}\right)>0$ for every $j$ 's. To construct such an example, we consider

$\mathrm{i} \partial_{t} u=\frac{\nu(E)}{2} H_{0} u+\left(\frac{a(E, \omega t)}{2} x^{2}-\frac{b(E, \omega t)}{2}\left(x \cdot \mathrm{i} \partial_{x}+\mathrm{i} \partial_{x} \cdot x\right)-\frac{c(E, \omega t)}{2} \partial_{x}^{2}\right) u$.

where $\nu(E)=\cos ^{-1}\left(-\frac{E}{2}\right), \mathcal{I} \subset[-2+\delta, 2-\delta]$ with $\delta$ a small numerical constant (e.g., $\left.\delta=10^{-6}\right)$. Then our result is the following:

Theorem 5. There exist a sub-interval $\mathcal{I} \subset[-2+\delta, 2-\delta]$ and $a, b, c:$ $\mathcal{I} \times \mathbb{T}^{2} \rightarrow \mathbb{R}$ with $a(E, \cdot), b(E, \cdot), c(E, \cdot) \in C^{\omega}\left(\mathbb{T}^{2}, \mathbb{R}\right)$ for every $E \in \mathcal{I}$, such that the conclusions of Theorem 1 and 2 hold for Eq. (5). Moreover, $\operatorname{Leb}\left(\Lambda_{j}\right)>0$ for every $j \in \mathbb{N}$.

Remark 1.3. One can even further get precise size of $\operatorname{Leb}\left(\Lambda_{j}\right)$ according to [30].

The rest of paper will be organised as follows. In Section 2 , which serves as a preliminary section, we recall the definition of Weyl quantization and some known results on the relation between classical Hamiltonian to quantum Hamiltonian. We give an abstract theorem in Section 3 on the reducibility for quantum Hamiltonian, provided that the reducibility for the corresponding classical Hamiltonian is known. By applying this abstract theorem, we 
exploit the connection between reducibility and property of Sobolev norm. The abstract theorem is proved in Section 4. In Section 5, we prove the main result just by verifying the hypothesis of abstract theorem. In Section 6, the proofs of Theorem $3-5$ are given.

\section{Classical Hamiltonian and quantum Hamiltonian}

To give some preliminary knowledge, let us recall the definition of Weyl quantization, which relates the classical and quantum mechanics, and its properties. The conclusions listed in this section can also be found in [7].

The Weyl quantization is the operator $\mathrm{Op}^{W}: f \mapsto f^{W}$ for any symbol $f=f(x, \xi)$, with $x, \xi \in \mathbb{R}^{n}$, where $f^{W}$ is the Weyl operator of $f$ :

$$
\left(f^{W} u\right)(x)=\frac{1}{(2 \pi)^{n}} \int_{y, \xi \in \mathbb{R}^{n}} e^{\mathrm{i}(x-y) \xi} f\left(\frac{x+y}{2}, \xi\right) u(y) d y d \xi, \quad \forall u \in L^{2}\left(\mathbb{R}^{n}\right) .
$$

In particular, if $f$ is a polynomial of degree at most 2 in $(x, \xi)$, then $f^{W}$ is a polynomial of degree at most 2 in $\left(x,-\mathrm{i} \partial_{x}\right)$ after the symmetrization.

For the 1-parameter family of Hamiltonian $\chi(t, x, \xi)$, with $t$ an external parameter, let $\phi^{\tau}(t, x, \xi)$ be the time $\tau$-flow it generates, precisely the solution of

$$
\frac{d x}{d \tau}=\frac{\partial \chi}{\partial \xi}(t, x, \xi), \quad \frac{d \xi}{d \tau}=-\frac{\partial \chi}{\partial x}(t, x, \xi) .
$$

The time-dependent coordinate transformation

$$
(x, \xi)=\phi^{1}(t, \tilde{x}, \tilde{\xi})=\left.\phi^{\tau}(t, \tilde{x}, \tilde{\xi})\right|_{\tau=1}
$$

transforms a Hamiltonian system with Hamiltonian $h$ into a system with Hamiltonian $g$ given by

$$
g(t, \tilde{x}, \tilde{\xi})=h\left(\phi^{1}(t, \tilde{x}, \tilde{\xi})\right)-\int_{0}^{1} \frac{\partial \chi}{\partial t}\left(t, \phi^{\tau}(t, \tilde{x}, \tilde{\xi})\right) d \tau
$$

Lemma 2.1 (Remark 2.6 of [7]). If the Weyl operator $\chi^{W}\left(t, x,-\mathrm{i} \partial_{x}\right)$ is self-adjoint for any fixed $t$, then the transformation

$$
\psi=e^{\mathrm{i} \chi^{W}\left(t, x,-\mathrm{i} \partial_{x}\right)} \tilde{\psi}
$$

transforms the equation $\mathrm{i} \partial_{t} \psi=H \psi$ into $\mathrm{i} \partial_{t} \tilde{\psi}=G \tilde{\psi}$ with

$$
\begin{aligned}
G:= & e^{\mathrm{i} \chi^{W}\left(t, x,-\mathrm{i} \partial_{x}\right)} H e^{-\mathrm{i} \chi^{W}\left(t, x,-\mathrm{i} \partial_{x}\right)} \\
& -\int_{0}^{1} e^{\mathrm{i} \tau \chi^{W}\left(t, x,-\mathrm{i} \partial_{x}\right)}\left(\partial_{t} \chi^{W}\left(t, x,-\mathrm{i} \partial_{x}\right)\right) e^{-\mathrm{i} \tau \chi^{W}\left(t, x,-\mathrm{i} \partial_{x}\right)} d \tau .
\end{aligned}
$$


Proposition 1 (Proposition 2.9 of [7]). Let $\chi(t, x, \xi)$ be a polynomial of degree at most 2 in $(x, \xi)$ with smooth time-dependent coefficients. If the transformation (6) transforms a classical system with Hamiltonian $h$ into a system with Hamiltonian $g$, then the transformation (7) transforms the quantum Hamiltonian system $h^{W}$ into $g^{W}$.

Now, let us focus on the case $n=1$.

Lemma 2.2 (Lemma 2.8 of [7]). Let $\chi(\theta, x, \xi)$ be a polynomial of degree at most 2 in $(x, \xi)$ with real coefficients depending in a $C^{\infty}$-way on $\theta \in \mathbb{T}^{d}$. For every $\theta \in \mathbb{T}^{d}$, the Weyl operator $\chi^{W}\left(\theta, x,-\mathrm{i} \partial_{x}\right)$ is self-adjoint in $L^{2}(\mathbb{R})$ and $e^{-\mathrm{i} \tau \chi^{W}\left(\theta, x,-\mathrm{i} \partial_{x}\right)}$ is unitary in $L^{2}\left(\mathbb{R}^{n}\right)$ for every $\tau \in \mathbb{R}$. Furthermore, if the coefficients of $\chi(\theta, x, \xi)$ are uniformly bounded w.r.t. $\theta \in \mathbb{T}^{d}$, then for any $s \geq 0$, there exist $c^{\prime}, C^{\prime}>0$ depending on $\left\|\left[H_{0}^{s}, \chi^{W}\left(\theta, x,-\mathrm{i} \partial_{x}\right)\right] H_{0}^{-s}\right\|_{L^{2} \mapsto L^{2}}$ and $s$, such that

$$
c^{\prime}\|\psi\|_{s} \leq\left\|e^{-\mathrm{i} \tau \chi^{W}\left(\theta, x,-\mathrm{i} \partial_{x}\right)} \psi\right\|_{s} \leq C^{\prime}\|\psi\|_{s}, \quad \tau \in[0,1], \quad \theta \in \mathbb{T}^{d} .
$$

\section{Reducibility and growth of Sobolev norm}

\subsection{An abstract theorem on reducibility}

Consider the 1-d time-dependent equation

$$
\mathrm{i} \partial_{t} u=L^{W}\left(\omega t, x,-\mathrm{i} \partial_{x}\right) u, \quad x \in \mathbb{R},
$$

where $L^{W}\left(\omega t, x,-\mathrm{i} \partial_{x}\right)$ is a linear differential operator, $\omega \in \mathbb{T}^{d}, d \geq 1$, and the symbol $L(\theta, x, \xi)$ is a quadratic form of $(x, \xi)$ with coefficients analytically depending on $\theta \in \mathbb{T}^{d}$. More precisely, we assume that

$$
L(\theta, x, \xi)=\frac{1}{2}\left(a(\theta) x^{2}+b(\theta) x \cdot \xi+b(\theta) \xi \cdot x+c(\theta) \xi^{2}\right),
$$

with coefficients $a, b, c \in C^{\omega}\left(\mathbb{T}^{d}, \mathbb{R}\right)$.

Through Weyl quantization, the reducibility for the time-dependent PDE can be related to the reducibility for the $\mathrm{sl}(2, \mathbb{R})$-linear system $(\omega, A(\cdot))$ :

$$
X^{\prime}=A(\omega t) X, \quad A \in C^{\omega}\left(\mathbb{T}^{d}, \operatorname{sl}(2, \mathbb{R})\right) .
$$

Given $A_{1}, A_{2} \in C^{\omega}\left(\mathbb{T}^{d}, \mathrm{sl}(2, \mathbb{R})\right)$, if there exists $Y \in C^{\omega}\left(2 \mathbb{T}^{d}, \mathrm{SL}(2, \mathbb{R})\right)$ such that

$$
\frac{d}{d t} Y(\omega t)=A_{1}(\omega t) Y(\omega t)-Y(\omega t) A_{2}(\omega t)
$$


we say that $\left(\omega, A_{1}(\cdot)\right)$ is conjugated to $\left(\omega, A_{2}(\cdot)\right)$ by $Y$. If $(\omega, A(\cdot))$ can be conjugated to $(\omega, B)$ with $B \in \operatorname{sl}(2, \mathbb{R})$, we say that $(\omega, A(\cdot))$ is reducible.

Now let $A(\cdot):=\left(\begin{array}{cc}b(\cdot) & c(\cdot) \\ -a(\cdot) & -b(\cdot)\end{array}\right) \in C^{\omega}\left(\mathbb{T}^{d}, \operatorname{sl}(2, \mathbb{R})\right)$ with $a, b, c$ coefficients given in (10).

Theorem 6. Assume that there exist $B \in \operatorname{sl}(2, \mathbb{R})$ and $Z_{j} \in C^{\omega}\left(2 \mathbb{T}^{d}, \operatorname{sl}(2, \mathbb{R})\right)$, $j=0, \cdots, K$, such that $(\omega, A(\cdot))$ is conjugated to $(\omega, B)$ by $\prod_{j=0}^{K} e^{Z_{j}}$. Then Eq. (9) is reducible, i.e., there exists a time quasi-periodic map $U(\omega t)$, unitary in $L^{2}$ and analytic on $t$, satisfying

$$
c^{\prime}\|\psi\|_{s} \leq\|U(\omega t) \psi\|_{s} \leq C^{\prime}\|\psi\|_{s}, \quad \forall \psi \in \mathcal{H}^{s},
$$

for constants $c^{\prime}, C^{\prime}>0$ depending on $s$ and $\psi$, such that $E q$. (9) is conjugated to

$$
\mathrm{i} \partial_{t} v=G v
$$

by the transformation $u=U(\omega t) v$, with $G$ an operator independent of time.

More precisely,

(I) $G$ is unitary equivalent to $\frac{\sqrt{\operatorname{det} B}}{2} H_{0}$ if

$$
\operatorname{det} B>0 \text { or } B=\left(\begin{array}{ll}
0 & 0 \\
0 & 0
\end{array}\right) \text {. }
$$

(II) $G$ is unitary equivalent to $-\frac{\mathrm{i} \sqrt{-\operatorname{det} B}}{2}\left(x \cdot \partial_{x}+\partial_{x} \cdot x\right)$ if

$$
\operatorname{det} B<0 \text {. }
$$

(III) $G$ is unitary equivalent to $-\frac{\kappa}{2} x^{2}$ if

$$
B \text { is similar to }\left(\begin{array}{ll}
0 & 0 \\
\kappa & 0
\end{array}\right) \text { with } \kappa \neq 0 \text {. }
$$

\subsection{Growth of Sobolev norm via reducibility}

As an corollary of Theorem 6, we have:

Theorem 7. Under the assumption of Theorem [6, we consider the solution $u(t)=u(t, \cdot)$ to Eq. (9) with the non-vanishing initial condition $u(0) \in \mathcal{H}^{s}$, $s \geq 0$. There exists $c, C>0$, depending on $s$ and $u(0)$, such that, for any $t \geq 0$, 
- If (19) holds, then $c \leq\|u(t)\|_{s} \leq C$.

- If (14) holds, then $c e^{\sqrt{-\operatorname{det} B s t}} \leq\|u(t)\|_{s} \leq C e^{\sqrt{-\operatorname{det} B} s t}$.

- If (15) holds, then $c|\kappa|^{s} t^{s} \leq\|u(t)\|_{s} \leq C|\kappa|^{s}{\sqrt{1+t^{2}}}^{\frac{s}{2}}$.

According to (11), to precise the growth of Sobolev norms for the solution to Eq. (9), it is sufficient to study the reduced quantum Hamiltonian $G\left(x,-\mathrm{i} \partial_{x}\right)$ obtained in (12), or more simply, the unitary equivalent forms of types (I)-(III) listed in Theorem 6 .

If (13) holds, then $G$ is unitary equivalent to $\frac{\sqrt{\operatorname{det} B}}{2} H_{0}$. Since the $\mathcal{H}^{s}-$ norm of $e^{-\mathrm{i} t \frac{\sqrt{\mathrm{det} B}}{2} H_{0}} \psi_{0}$ is conserved for any $\psi_{0} \in \mathcal{H}^{s}$, the boundedness of Sobolev norm is shown. We focus on the cases where (14) and (15) hold, in which the growth of Sobolev norm occurs.

Proposition 2. For the equation

$$
\partial_{t} v(t, x)=-\frac{\lambda}{2} x \cdot \partial_{x} v(t, x)-\frac{\lambda}{2} \partial_{x}(x \cdot v(t, x)), \quad \lambda>0,
$$

with non-vanishing initial condition $v(0, \cdot)=v_{0} \in \mathcal{H}^{s}, s \geq 0$, there exist two constants $\tilde{c}, \tilde{C}>0$, depending on $s, \lambda$ and $v_{0}$, such that the solution satisfies

$$
\tilde{c} e^{\lambda s t} \leq\|\psi(t, \cdot)\|_{s} \leq \tilde{C} e^{\lambda s t}, \quad \forall t \geq 0 .
$$

Remark 3.1. This conclusion is also given in Remark 1.4 of [34].

Proof: Through a straightforward computation, we can verify that, for the initial condition $v(0, \cdot)=v_{0}(\cdot) \in \mathcal{H}^{s}$, the solution to Eq. (16) satisfies

$$
v(t, x)=e^{-\frac{\lambda}{2} t} v_{0}\left(e^{-\lambda t} x\right) .
$$

For any $s \geq 0$,

$$
\begin{aligned}
\int_{\mathbb{R}} x^{2 s}|v(t, x)|^{2} d x & =\int_{\mathbb{R}} x^{2 s}\left|v_{0}\left(e^{-\lambda t} x\right)\right|^{2} d\left(e^{-\lambda t} x\right) \\
& =e^{2 \lambda s t} \int_{\mathbb{R}}\left(e^{-\lambda t} x\right)^{2 s}\left|v_{0}\left(e^{-\lambda t} x\right)\right|^{2} d\left(e^{-\lambda t} x\right) \\
& =e^{2 \lambda s t} \int_{\mathbb{R}} x^{2 s}\left|v_{0}(x)\right|^{2} d x .
\end{aligned}
$$


and for $s \in \mathbb{N}$,

$$
\int_{\mathbb{R}}\left|\partial_{x}^{s} v(t, x)\right|^{2} d x=e^{-2 \lambda s t} \int_{\mathbb{R}}\left|v_{0}^{(s)}\left(e^{-\lambda t} x\right)\right|^{2} d\left(e^{-\lambda t} x\right)=e^{-2 \lambda s t} \int_{\mathbb{R}}\left|v_{0}^{(s)}(x)\right|^{2} d x .
$$

In view of the equivalent definition (2) of the $\mathcal{H}^{s}$-norm given in Remark 1.1, we get (17) by combining (18) and (19).

Proposition 3. For the equation

$$
\mathrm{i} \partial_{t} v(t, x)=-\frac{\kappa}{2} x^{2} \cdot v(t, x), \quad \kappa \in \mathbb{R},
$$

with non-vanishing initial condition $v_{0} \in \mathcal{H}^{s}, s \geq 0$, there exists constants $\tilde{c}, \tilde{C}>0$, depending on $s, \kappa$ and $v_{0}$, such that the solution satisfies

$$
\tilde{c}|\kappa|^{s}|t|^{s} \leq\|v(t, \cdot)\|_{s} \leq \tilde{C}|\kappa|^{s}\left(1+t^{2}\right)^{\frac{s}{2}}, \quad \forall t \in \mathbb{R} .
$$

Proof: With the initial condition $v(0, \cdot)=v_{0}(\cdot) \in \mathcal{H}^{s}$, the solution to Eq. (20) is

$$
v(t, x)=e^{\mathrm{i} \frac{\kappa}{2} x^{2} t} v_{0}(x)
$$

For any $s \geq 0$,

$$
\left\|x^{s} v(t, x)\right\|_{L^{2}}=\left\|x^{s} e^{\mathrm{i} \frac{\kappa}{2} x^{2} t} v_{0}(x)\right\|_{L^{2}}=\left\|x^{s} v_{0}(x)\right\|_{L^{2}}
$$

and for $s \in \mathbb{N}$,

$$
\begin{aligned}
\partial_{x}^{s}(v(t, x))= & \partial_{x}^{s}\left(e^{\mathrm{i} \frac{\kappa}{2} x^{2} t} v_{0}(x)\right) \\
= & \sum_{\alpha=0}^{s} C_{s}^{\alpha}\left(e^{\mathrm{i} \frac{\kappa}{2} x^{2} t}\right)^{(\alpha)} v_{0}^{(s-\alpha)}(x) \\
= & e^{\mathrm{i} \frac{\kappa}{2} x^{2} t} \sum_{\alpha=0}^{s} C_{s}^{\alpha}\left((\mathrm{i} \kappa t)^{\alpha} x^{\alpha}+P_{\alpha}(\mathrm{i} \kappa t, x)\right) v_{0}^{(s-\alpha)}(x) \\
= & (\mathrm{i} \kappa t)^{s} x^{s} e^{\mathrm{i} \frac{\kappa}{2} x^{2} t} \cdot v_{0}(x)+P_{s}(\mathrm{i} \kappa t, x) e^{\mathrm{i} \frac{\kappa}{2} x^{2} t} \cdot v_{0}(x) \\
& +x^{\alpha} e^{\mathrm{i} \frac{\kappa}{2} x^{2} t} \sum_{\alpha=0}^{s-1} C_{s}^{\alpha}\left((\mathrm{i} \kappa t)^{\alpha} x^{\alpha}+P_{\alpha}(\mathrm{i} \kappa t, x)\right) v_{0}^{(s-\alpha)}(x),
\end{aligned}
$$

where, for $\alpha \geq 2, P_{\alpha}(\mathrm{i} \kappa t, x)$ is a polynomial of degree $\alpha-2$ of $x$, with the coefficients being monomials of i $\kappa t$ of degree $\leq \alpha-1$ and $P_{1}=P_{0}=0$. Then, there exists a constant $D>0$ such that

$$
\left.\left.\left|\left\|\partial_{x}^{s}(v(t, x))\right\|_{L^{2}}-\right| \kappa t\right|^{s}\left\|x^{s} v_{0}(x)\right\|_{L^{2}}|\leq D| \kappa t\right|^{s-1}\left\|v_{0}(x)\right\|_{s}
$$


In view of the equivalent definition (2) of norm in Remark 1.1, we get (21).

Proof of Theorem 7. From Theorem 6, we know that Eq. (9) is conjugated to $\mathrm{i} \partial_{t} v=G v$ by the transformation $u=U(\omega t) v$, with $G=G\left(x,-\mathrm{i} \partial_{x}\right)$ the operator independent of $t$ given in (25).

Recall Proposition 2 and 3. Given $s \geq 0$, for any non-vanishing $v_{0} \in \mathcal{H}^{s}$, for the three types of unitary equivalence of $G$, there are three different behaviours of the solution to the equation $\mathrm{i} \partial_{t} v=G v$ as $t \rightarrow \infty$.

- If $G$ is unitary equivalent to $\frac{\sqrt{\operatorname{det} B}}{2} H_{0}$ (under (13) ), then $\left\|e^{-\mathrm{i} G t} v_{0}\right\|_{s}=$ $O(1)$.

- If $G$ is unitary equivalent to $-\frac{\mathrm{i} \sqrt{-\operatorname{det} B}}{2}\left(x \cdot \partial_{x}+\partial_{x} \cdot x\right)$ (under (14)), then $\left\|e^{-\mathrm{i} G t} v_{0}\right\|_{s}=O\left(e^{\sqrt{-\operatorname{det} B} s t}\right)$.

- If $G$ is unitary equivalent to $-\frac{\kappa}{2} x^{2}$ (under (15)), then $\left\|e^{-\mathrm{i} G t} v_{0}\right\|_{s}=$ $O\left(|\kappa|^{s} t^{s}\right)$.

Moreover, according to (11), for $s \geq 0$, there exist constants $c^{\prime}, C^{\prime}>0$ such that

$$
c^{\prime}\|v\|_{s} \leq\|U(\omega t) v\|_{s} \leq C^{\prime}\|v\|_{s}, \quad \forall v \in \mathcal{H}^{s} .
$$

Hence Theorem 7 is shown.

\section{Reducibility in classical Hamiltonian system and proof of The- orem 6}

\subsection{Conjugation between classical hamiltonians}

Given two quadratic classical Hamiltonians

$$
h_{j}(\omega t, x, \xi)=\frac{1}{2}\left(a_{j}(\omega t) x^{2}+2 b_{j}(\omega t) x \cdot \xi+c_{j}(\omega t) \xi^{2}\right), \quad j=1,2,
$$

which can be presented as

$$
h_{j}(\omega t, x, \xi)=\frac{1}{2}\left(\begin{array}{c}
x \\
\xi
\end{array}\right)^{\top} J A_{j}(\omega t)\left(\begin{array}{c}
x \\
\xi
\end{array}\right), \quad j=1,2
$$

with $J:=\left(\begin{array}{cc}0 & -1 \\ 1 & 0\end{array}\right)$ and $A_{j}(\cdot)=\left(\begin{array}{cc}b_{j}(\cdot) & c_{j}(\cdot) \\ -a_{j}(\cdot) & -b_{j}(\cdot)\end{array}\right) \in C^{\omega}\left(\mathbb{T}^{d}, \operatorname{sl}(2, \mathbb{R})\right)$. The corresponding equations of motion are given by

$$
x^{\prime}=\frac{\partial h_{j}}{\partial \xi}, \quad \xi^{\prime}=-\frac{\partial h_{j}}{\partial x}, \quad j=1,2,
$$


which are the linear systems $\left(\omega, A_{j}\right)$ :

$$
\left(\begin{array}{l}
x(t) \\
\xi(t)
\end{array}\right)^{\prime}=A_{j}(\omega t)\left(\begin{array}{l}
x(t) \\
\xi(t)
\end{array}\right) .
$$

Proposition 4. If the linear system $\left(\omega, A_{1}(\cdot)\right)$ is conjugated to $\left(\omega, A_{2}(\cdot)\right)$ by a time quasi-periodic $\mathrm{SL}(2, \mathbb{R})$-transformation, i.e.,

$$
\frac{d}{d t} e^{Z(\omega t)}=A_{1}(\omega t) e^{Z(\omega t)}-e^{Z(\omega t)} A_{2}(\omega t), \quad Z \in C^{\omega}\left(2 \mathbb{T}^{d}, \operatorname{sl}(2, \mathbb{R})\right),
$$

then the classical Hamiltonian $h_{1}(\omega t, x, \xi)$ is conjugated to $h_{2}(\omega t, x, \xi)$ via the time-1 flow $\phi_{\chi}^{1}(t, x, \xi)$ generated by the Hamiltonian

$$
\chi(\omega t, x, \xi)=\frac{1}{2}\left(\begin{array}{c}
x \\
\xi
\end{array}\right)^{\top} J Z(\omega t)\left(\begin{array}{c}
x \\
\xi
\end{array}\right) .
$$

Remark 4.1. This is actually a consequence of the fact that in dimension 2(one degree of freedom), canonical transformations coincide with the transformations which preserve the volume.

Proof: Note that the equation of motion of the classical Hamiltonian $h_{1}$ is the linear system $\left(\omega, A_{1}(\cdot)\right)$ :

$$
\left(\begin{array}{l}
x \\
\xi
\end{array}\right)^{\prime}=A_{1}(\omega t)\left(\begin{array}{l}
x \\
\xi
\end{array}\right) \text {. }
$$

In view of (22), the transformation

$$
\left(\begin{array}{c}
x \\
\xi
\end{array}\right)=e^{Z(\omega t)}\left(\begin{array}{c}
\tilde{x} \\
\tilde{\xi}
\end{array}\right), \quad Z \in C^{\omega}\left(2 \mathbb{T}^{d}, \mathrm{sl}(2, \mathbb{R})\right),
$$

conjugates $\left(\omega, A_{1}(\cdot)\right)$ to $\left(\omega, A_{2}(\cdot)\right)$. More precisely,

$$
\begin{aligned}
\left(\begin{array}{c}
\tilde{x} \\
\tilde{\xi}
\end{array}\right)^{\prime} & =e^{-Z(\omega t)} A_{1}(\omega t)\left(\begin{array}{c}
x \\
\xi
\end{array}\right)-e^{-Z(\omega t)} \frac{d}{d t} e^{Z(\omega t)}\left(\begin{array}{c}
\tilde{x} \\
\tilde{\xi}
\end{array}\right) \\
& =e^{-Z(\omega t)} A_{1}(\omega t) e^{Z(\omega t)}\left(\begin{array}{c}
\tilde{x} \\
\tilde{\xi}
\end{array}\right)-e^{-Z(\omega t)} \frac{d}{d t} e^{Z(\omega t)}\left(\begin{array}{c}
\tilde{x} \\
\tilde{\xi}
\end{array}\right) \\
& =A_{2}(\omega t)\left(\begin{array}{c}
\tilde{x} \\
\tilde{\xi}
\end{array}\right),
\end{aligned}
$$

for which the corresponding Hamiltonian is $h_{2}(\omega t, \tilde{x}, \tilde{\xi})$. As in (3-35) of [7], the time-1 map between the two Hamiltonians is generated by (23) since there is only quadratic terms in the Hamiltonian in our case. 


\subsection{Proof of Theorem [}

We consider the classical Hamiltonian

$$
\begin{aligned}
L(\omega t, x, \xi) & =\frac{a(\omega t)}{2} x^{2}+\frac{b(\omega t)}{2}(x \cdot \xi+\xi \cdot x)+\frac{c(\omega t)}{2} \xi^{2} \\
& =\frac{1}{2} X^{\top} J A(\omega t) X, \quad X:=\left(\begin{array}{c}
x \\
\xi
\end{array}\right) .
\end{aligned}
$$

with $a, b, c \in C^{\omega}\left(\mathbb{T}^{d}\right)$ given in Eq. (9) , and $A:=\left(\begin{array}{cc}b & c \\ -a & -b\end{array}\right) \in C^{\omega}\left(\mathbb{T}^{d}, \operatorname{sl}(2, \mathbb{R})\right)$.

By the hypothesis of Theorem 6 , the linear system $(\omega, A(\cdot))$ can be reduced to the constant system $(\omega, B)$, with $B=\left(\begin{array}{cc}B_{11} & B_{12} \\ -B_{21} & -B_{11}\end{array}\right) \in \mathrm{sl}(2, \mathbb{R})$, via finitely many transformations $\left(e^{Z_{j}}\right)_{j=0}^{K}$ with $Z_{j} \in C^{\omega}\left(2 \mathbb{T}^{d}, \mathrm{sl}(2, \mathbb{R})\right)$. Hence the reduced classical Hamiltonian is

$$
g(x, \xi)=\frac{1}{2} X^{\top} J B X=\frac{B_{21}}{2} x^{2}+\frac{B_{11}}{2}(x \cdot \xi+\xi \cdot x)+\frac{B_{12}}{2} \xi^{2} .
$$

By Proposition 1, we see that $L^{W}\left(\omega t, x,-\mathrm{i} \partial_{x}\right)$ is conjugated to

$$
G\left(x,-\mathrm{i} \partial_{x}\right):=g^{W}\left(x,-\mathrm{i} \partial_{x}\right)=\frac{B_{21}}{2} x^{2}-\frac{B_{11}}{2}\left(x \cdot \mathrm{i} \partial_{x}+\mathrm{i} \partial_{x} \cdot x\right)-\frac{B_{12}}{2} \partial_{x}^{2}
$$

via the product of unitary (in $L^{2}(\mathbb{R})$ ) transformations

$$
U(\omega t):=\prod_{j=0}^{K} e^{-\mathrm{i} \chi_{j}^{W}\left(\omega t, x,-\mathrm{i} \partial_{x}\right)}
$$

where $\chi_{j}^{W}$ is the Weyl quantization of

$$
\chi_{j}(\omega t, x, \xi)=\frac{1}{2} X^{\top} J Z_{j}(\omega t) X .
$$

Then (11) is deduced from (8) in Lemma 2.2. The following diagram gives a 
straightforward explanation for the above proof.

$$
\begin{aligned}
& X^{\prime}=A(\omega t) X \quad \stackrel{\prod_{j=0}^{K} e^{Z_{j}(\omega t)}}{\longrightarrow} \quad X^{\prime}=B X \quad Z_{j} \in C^{\omega}\left(2 \mathbb{T}^{d}, \operatorname{sl}(2, \mathbb{R})\right) \\
& \uparrow \quad \uparrow \\
& L(\omega t)=\frac{1}{2} X^{\top} J A(\omega t) X \quad \Phi_{\chi_{0}(\omega t)}^{1} \stackrel{\circ \cdots \circ \Phi_{\chi_{K}(\omega t)}^{1}}{\longrightarrow} g=\frac{1}{2} X^{\top} J B X \quad \chi_{j}=\frac{1}{2} X^{\top} J Z_{j} X \\
& \uparrow \quad \uparrow \\
& \mathrm{i} \partial_{t} u=L^{W}(\omega t) u \quad \prod_{j=0}^{K} \stackrel{e^{-i} \chi_{j}^{W}(\omega t)}{\longrightarrow} \quad \mathrm{i} \partial_{t} u=g^{W} u \\
& B=e^{C_{B}}\left(\begin{array}{cc}
0 & \sqrt{\operatorname{det} B} \\
-\sqrt{\operatorname{det} B} & 0
\end{array}\right) e^{-C_{B}} .
\end{aligned}
$$

If (14) holds, i.e., $\operatorname{det} B<0$, then there exists $C_{B} \in \operatorname{sl}(2, \mathbb{R})$ such that

$$
B=e^{C_{B}}\left(\begin{array}{cc}
\sqrt{-\operatorname{det} B} & 0 \\
0 & -\sqrt{-\operatorname{det} B}
\end{array}\right) e^{-C_{B}} .
$$

If (15) holds, then there exists $C_{B} \in \operatorname{sl}(2, \mathbb{R})$ such that

$$
B=e^{C_{B}}\left(\begin{array}{cc}
0 & 0 \\
\kappa & 0
\end{array}\right) e^{-C_{B}}
$$

Therefore, for Eq. (91), the three types of unitary equivalence of $G=$ $G\left(x,-\mathrm{i} \partial_{x}\right)$ are shown by $(26)-(28)$ respectively.

\section{Proof of Theorem 1 and 2}

In view of Theorem 6, to show the reducibility of Eq. (1), it is sufficient to show the reducibility of the corresponding $\operatorname{sl}(2, \mathbb{R})$-linear system.

For $E \in \mathcal{I}$, the symbol of the quantum Hamiltonian (1) is

$$
h_{E}(\omega t, x, \xi)=\frac{\nu(E)}{2}\left(\xi^{2}+x^{2}\right)+W(E, \omega t, x, \xi)
$$


which corresponds the quasi-periodic linear system $\left(\omega, A_{0}+F_{0}\right)$

$$
\left(\begin{array}{l}
x \\
\xi
\end{array}\right)^{\prime}=\left[\left(\begin{array}{cc}
0 & \nu(E) \\
-\nu(E) & 0
\end{array}\right)+\left(\begin{array}{cc}
b(E, \omega t) & c(E, \omega t) \\
-a(E, \omega t) & -b(E, \omega t)
\end{array}\right)\right]\left(\begin{array}{l}
x \\
\xi
\end{array}\right)
$$

where, for every $E \in \mathcal{I}$,

$$
\begin{aligned}
A_{0}(E) & :=\left(\begin{array}{cc}
0 & \nu(E) \\
-\nu(E) & 0
\end{array}\right) \in \operatorname{sl}(2, \mathbb{R}), \\
F_{0}(E, \cdot) & :=\left(\begin{array}{cc}
b(E, \cdot) & c(E, \cdot) \\
-a(E, \cdot) & -b(E, \cdot)
\end{array}\right) \in C_{r}^{\omega}\left(\mathbb{T}^{d}, \operatorname{sl}(2, \mathbb{R})\right)
\end{aligned}
$$

with $\left|\partial_{E}^{m} F_{0}\right|_{r}<\varepsilon_{0}, m=0,1,2$, sufficiently small.

The reducibility of linear system (29) was proved by Eliasson [14] (see also [25] for results about $\operatorname{SL}(2, \mathbb{R})$-cocycles). We summarise the needed results in the following proposition. To make the paper as self-contained as possible, we give a short proof without adding too many details on known facts. Since every quantity depends on $E$, we do not always write this dependence explicitly in the statement of proposition.

Before stating the precise result, we introduce the concept of rotation number. The rotation number of quasi-periodic $\operatorname{sl}(2, \mathbb{R})$-linear system (29) is defined as

$$
\rho(E)=\rho\left(\omega, A_{0}(E)+F(E, \omega t)\right)=\lim _{t \rightarrow \infty} \frac{\arg \left(\Phi_{E}^{t} X\right)}{t}, \quad \forall X \in \mathbb{R}^{2} \backslash\{0\},
$$

where $\Phi_{E}^{t}$ is the basic matrix solution and $\arg$ denotes the angle. The rotation number $\rho$ is well-defined and it does not depend on $X$ [28].

Proposition 5. There exists $\varepsilon_{*}=\varepsilon_{*}\left(r, \gamma, \tau, d, l_{1}, l_{2}\right)>0$ such that if

$$
\max _{m=0,1,2}\left|\partial_{E}^{m} F_{0}\right|_{r}=: \varepsilon_{0}<\varepsilon_{*},
$$

then the following holds for the quasi-periodic linear system $\left(\omega, A_{0}+F_{0}\right)$.

(1) For a.e. $E \in \mathcal{I},\left(\omega, A_{0}+F_{0}(\cdot)\right)$ is reducible. More precisely, there exist $B \in \operatorname{sl}(2, \mathbb{R})$ and $Z_{j} \in C^{\omega}\left(2 \mathbb{T}^{d}, \operatorname{sl}(2, \mathbb{R})\right), j=0,1, \cdots, K$, such that

$$
\frac{d}{d t}\left(\prod_{j=0}^{K} e^{Z_{j}(\omega t)}\right)=\left(A_{0}+F_{0}(\omega t)\right)\left(\prod_{j=0}^{K} e^{Z_{j}(\omega t)}\right)-\left(\prod_{j=0}^{K} e^{Z_{j}(\omega t)}\right) B
$$


(2) The rotation number $\rho=\rho(E)$ is monotonic on $\mathcal{I}$. For any $k \in \mathbb{Z}^{d}$,

$$
\tilde{\Lambda}_{k}:=\left\{E \in \overline{\mathcal{I}}: \rho(E)=\frac{\langle k, \omega\rangle}{2}\right\} \text { 卢 }
$$

is a closed interval, and we have

$$
\sum_{k \in \mathbb{Z}^{d}} \operatorname{Leb}\left(\tilde{\Lambda}_{k}\right)<\varepsilon_{0}^{\frac{1}{40}} .
$$

(3) For every $E \in \tilde{\Lambda}_{k}=:\left[a_{k}, b_{k}\right],\left(\omega, A_{0}+F_{0}(\cdot)\right)$ is reducible and the matrix $B \in \operatorname{sl}(2, \mathbb{R})$ in (31) satisfies

- if $a_{k}=b_{k}$, then $B=\left(\begin{array}{ll}0 & 0 \\ 0 & 0\end{array}\right)$;

- if $a_{k}<b_{k}$, then

$-\operatorname{det} B<0$ for $E \in\left(a_{k}, b_{k}\right)$,

$-\operatorname{det} B=0$ for $E=a_{k}, b_{k}$ and $E \notin \partial \mathcal{I}$.

(4) For a.e. $E \in \mathcal{I} \backslash \bigcup_{k} \tilde{\Lambda}_{k},\left(\omega, A_{0}+F_{0}(\cdot)\right)$ is reducible and the matrix $B \in \operatorname{sl}(2, \mathbb{R})$ in (31) satisfies $\operatorname{det} B>0$.

Proof: Since $\nu$ is a strictly monotonic real-valued function of $E \in \mathcal{I}$ and $\left|\nu^{\prime}\right| \geq l_{1},\left|\nu^{\prime \prime}\right| \leq l_{2}$, (30) implies that $\left|\partial_{E}^{m} F_{0}\left(\nu^{-1}(E), \cdot\right)\right|_{r}, m=0,1,2$, is also small enough. Hence, to prove the above arguments, we can simply consider the case where $\nu(E)=E \in \mathcal{I}=\mathbb{R}$ and then obtain Proposition 5 by replacing $E$ by $\nu(E)$.

Proof of (1). The reducibility has already been shown by Eliasson [14] for a.e. $E \in \mathbb{R}$. Indeed, if $\max _{m=0,1,2}\left|\partial_{E}^{m} F_{0}\right|_{r}$ is small enough (depending on $r, \gamma, \tau, d$ ), then there exists sequences $\left(Y_{j}\right)_{j \in \mathbb{N}} \subset C^{\omega}\left(2 \mathbb{T}^{d}, \mathrm{SL}(2, \mathbb{R})\right),\left(A_{j}\right)_{j \in \mathbb{N}} \subset \operatorname{sl}(2, \mathbb{R})$, and $\left(F_{j}\right)_{j \in \mathbb{N}} \subset C^{\omega}\left(2 \mathbb{T}^{d}, \mathrm{sl}(2, \mathbb{R})\right)$, all of which are piecewise $C^{2}$ w.r.t. $E$, with $\max _{m=0,1,2}\left|\partial_{E}^{m} F_{j}\right|_{\mathbb{T}^{d}}<\varepsilon_{j}:=\varepsilon_{0}^{(1+\sigma)^{j}}$ for $\sigma=\frac{1}{33}$, such that

$$
\frac{d}{d t} Y_{j}(\omega t)=\left(A_{j}+F_{j}(\omega t)\right) Y_{j}(\omega t)-Y_{j}(\omega t)\left(A_{j+1}+F_{j+1}(\omega t)\right) .
$$

\footnotetext{
${ }^{5} \tilde{\Lambda}_{k}$ can be empty for some $k \in \mathbb{Z}^{d}$ if the closed interval $\rho^{-1}\left(\frac{\langle k, \omega\rangle}{2}\right)$ does not intersect $\mathcal{I}$.
} 
More precisely, at the $j$-th step, for $\pm \mathrm{i} \xi_{j} \in \mathbb{R} \cup \mathrm{i} \mathbb{R}$, the two eigenvalues of $A_{j}$, and

$$
N_{j}:=\frac{2 \sigma}{r_{j}-r_{j+1}} \ln \left(\frac{1}{\varepsilon_{j}}\right)
$$

with $\left(r_{j}\right)_{j \in \mathbb{N}}$ a decreasing sequence of positive numbers such that $r_{j}-r_{j+1} \geq$ $2^{-(j+1)} r$ for each $j$,

- (non-resonant case) if for every $n \in \mathbb{Z}^{d}$ with $0<|n| \leq N_{j}$, we have

$$
\left|2 \xi_{j}-\langle n, \omega\rangle\right| \geq \varepsilon_{j}^{\sigma},
$$

then $Y_{j}=e^{\tilde{Z}_{j}}$ for some $\tilde{Z}_{j} \in C^{\omega}\left(2 \mathbb{T}^{d}, \operatorname{sl}(2, \mathbb{R})\right)$ with $\left|\tilde{Z}_{j}\right|_{2 \mathbb{T}^{d}}<\varepsilon_{j}^{\frac{2}{3}}$, and $\left|A_{j+1}-A_{j}\right|<\varepsilon_{j}^{\frac{2}{3}}$;

- (resonant) if for some $n_{j} \in \mathbb{Z}^{d}$ with $0<\left|n_{j}\right| \leq N_{j}$, we have

$$
\left|2 \xi_{j}-\left\langle n_{j}, \omega\right\rangle\right|<\varepsilon_{j}^{\sigma},
$$

$$
\begin{aligned}
& \text { then } Y_{j+1}(\cdot)=e^{\frac{\left\langle n_{j}, \dot{\gamma}\right.}{2 \xi_{j}} A_{j}} e^{\tilde{Z}_{j+1}} \text { for some } \tilde{Z}_{j} \in C^{\omega}\left(2 \mathbb{T}^{d}, \text { sl }(2, \mathbb{R})\right) \text { with }\left|\tilde{Z}_{j}\right|_{2 \mathbb{T}^{d}}< \\
& \varepsilon_{j}^{\frac{2}{3}} \text { and }\left|A_{j+1}\right|<\varepsilon_{j}^{\frac{\sigma}{2}} .
\end{aligned}
$$

As $j$ goes to $\infty$, the time-dependent part $F_{j}$ tends to vanish. For the detailed proof, we can refer to Lemma 2 of [14] and its proof.

In view of Lemma $3 \mathrm{~b})$ of [14], if the rotation number $\rho(E)$ of $\left(\omega, A_{0}(E)+\right.$ $F_{0}$ ) is Diophantine or rational w.r.t. $\omega$, which corresponds to a.e. $E \in \mathbb{R}$, then the resonant case occurs for only finitely many times. Therefore, for a.e. $E \in \mathbb{R}$, there exists a large enough $J_{*} \in \mathbb{N}^{*}$, depending on $E$, such that

$$
Y_{j}=e^{\tilde{Z}_{j}} \text { with }\left|\tilde{Z}_{j}\right|_{2 \mathbb{T}^{d}}<\varepsilon_{j}^{\frac{2}{3}}, \quad \forall j \geq J_{*} .
$$

This implies that $\prod_{j=0}^{\infty}\left|Y_{j}\right|_{2 \mathbb{T}^{d}}$ is convergent. As explained in the proof of Lemma 3.5 of [7], (35) also implies that there exists $S \in C^{\omega}\left(2 \mathbb{T}^{d}, \operatorname{sl}(2, \mathbb{R})\right)$ such that $\prod_{j=J_{*}}^{\infty} Y_{j}=e^{S}$, since $\varepsilon_{0}$ is sufficiently small. Hence (31) is shown, i.e., the reducibility is realized via finitely many transformations of the form $e^{Z_{j}(\omega t)}$ with $Z_{j} \in C^{\omega}\left(2 \mathbb{T}^{d}, \operatorname{sl}(2, \mathbb{R})\right)$.

Proof of (2). For $k \in \mathbb{Z}^{d}, \tilde{\Lambda}_{k}$ is obtained after several resonant KAM-steps, saying $j_{1}, \cdots, j_{L}$, where $n_{j_{i}} \in \mathbb{Z}^{d}$ with $0<\left|n_{j_{i}}\right| \leq N_{j_{i}}, i=1, \cdots, L$, satisfies

$$
\left|2 \xi_{j_{i}}-\left\langle n_{j_{i}}, \omega\right\rangle\right|<\varepsilon_{j_{i}}^{\sigma},
$$


and $k=n_{j_{1}}+\cdots+n_{j_{L}}$. We will show that

$$
\frac{10\left|n_{j_{L}}\right|}{11} \leq|k| \leq \frac{12\left|n_{j_{L}}\right|}{11}
$$

Assume that $L \geq 2$ (otherwise we have already $\left.k=n_{j_{L}}\right)$. After the $\left(j_{i-1}+\right.$ 1)-th step, $i=2, \cdots, L$, the eigenvalues $\pm \mathrm{i} \xi_{j_{i-1}+1}$ satisfies $\left|\xi_{j_{i-1}+1}\right|<2 \varepsilon_{j_{i-1}}^{\frac{\sigma}{2}}$. On the other hand, before the $\left(j_{i}+1\right)$-th step, the resonant condition (34) implies that the eigenvalues $\pm \mathrm{i} \xi_{j_{L}}$ satisfy that

$$
\left|2 \xi_{j_{i}}-\left\langle n_{j_{i}}, \omega\right\rangle\right| \leq \varepsilon_{j_{i}}^{\sigma} .
$$

Since the steps between these two successive resonant steps are all nonresonant, and $\omega \in \mathrm{DC}_{d}(\gamma, \tau)$, we have that

$$
\frac{\gamma}{\left|n_{j_{i}}\right|^{\tau}} \leq\left|\left\langle n_{j_{i}}, \omega\right\rangle\right| \leq 2\left|\xi_{j_{i-1}+1}\right|+2 \varepsilon_{j_{i-1}+1}^{\frac{1}{3}}+\varepsilon_{j_{i}}^{\sigma}<3 \varepsilon_{j_{i-1}}^{\frac{\sigma}{2}},
$$

which implies that

$$
\left|n_{j_{i}}\right|>\left(\frac{\gamma}{3}\right)^{\frac{1}{\tau}} \varepsilon_{j_{i-1}}^{-\frac{\sigma}{2 \tau}}>12\left|N_{j_{i-1}}\right| \geq 12\left|n_{j_{i-1}}\right| .
$$

Hence, we get (36).

$\tilde{\Lambda}_{k}$ is firstly formed at the $j_{L}$-th step, with the initial measure smaller than $\varepsilon_{j_{L}}^{2 \sigma}$. Since all the succedent steps are non-resonant, the measure of $\tilde{\Lambda}_{k}$ varies up to $\varepsilon_{j_{L}}^{2 \sigma}$. Then, for $\varsigma:=\frac{\ln (1+\sigma)}{\ln (8+8 \sigma)}$, we have

$$
\operatorname{Leb}\left(\tilde{\Lambda}_{k}\right)<2 \varepsilon_{j_{L}}^{2 \sigma}<2 \varepsilon_{0}^{\sigma} e^{-\left(\frac{12}{11}\right)^{\varsigma} N_{j_{L}}^{\varsigma}} \leq 2 \varepsilon_{0}^{\sigma} e^{-\left(\frac{12}{11}\right)^{\varsigma}\left|n_{j_{L}}\right|^{\varsigma}} .
$$

Indeed, recalling that $r_{j}-r_{j+1} \geq 2^{-(j+1)} r$ for every $j$, we have

$$
\begin{aligned}
\varepsilon_{j_{L}} & =\exp \left\{-\left|\ln \varepsilon_{0}\right|(1+\sigma)^{j_{L}}\right\} \\
& =\exp \left\{-\frac{\left|\ln \varepsilon_{0}\right|^{1-\varsigma}(1+\sigma)^{j_{L}(1-\varsigma)}\left(r_{j_{L}}-r_{j_{L}+1}\right)^{\varsigma}}{(2 \sigma)^{\varsigma}} N_{j_{L}}^{\varsigma}\right\} \\
& \leq \exp \left\{-\frac{\left|\ln \varepsilon_{0}\right|^{1-\varsigma} r^{\varsigma}}{(4 \sigma)^{\varsigma}}\left(\frac{(1+\sigma)^{1-\varsigma}}{2^{\varsigma}}\right)^{j_{L}} N_{j_{L}}^{\varsigma}\right\} \\
& <\exp \left\{-\left(\frac{12}{11}\right)^{\varsigma} \frac{N_{j_{L}}^{\varsigma}}{\sigma}\right\},
\end{aligned}
$$


since $\varepsilon_{0}$ is small enough and

$$
\frac{(1+\sigma)^{1-\varsigma}}{2^{\varsigma}}=\exp \left\{\frac{\ln (1+\sigma)}{\ln (8+8 \sigma)}(\ln 8-\ln 2)\right\}>1 .
$$

Therefore, by (36), we get $\operatorname{Leb}\left(\tilde{\Lambda}_{k}\right)<2 \varepsilon_{0}^{\sigma} e^{-|k|^{\varsigma}}$, which implies (32). For detailed proof of the measure estimate of $\tilde{\Lambda}_{k}$, we can also refer to Corollary 1 of [25].

Proof of (3) and (4). It can be deduced from Lemma 5 of [14].

Proof of Theorem 1 and 2. Theorem 2 can be seen as a corollary of Theorem 7. According to Theorem 6, the reducibility of Eq. (1) for a.e. $E \in \mathcal{I}$ is deduced from Proposition 5-(1). Let $\left\{\Lambda_{j}\right\}_{j \in \mathbb{N}}$ be the intervals $\tilde{\Lambda}_{k}$ 's intersecting $\mathcal{I}$ and let

$$
\mathcal{O}_{\varepsilon_{0}}:=\bigcup_{j \in \mathbb{N}} \Lambda_{j}=\bigcup_{k \in \mathbb{Z}^{d}} \tilde{\Lambda}_{k}
$$

Proposition 5 - $(2)$ gives the measure estimate of $\mathcal{O}_{\varepsilon_{0}}$. The unitary equivalences of the reduced quantum Hamiltonian follow from Proposition 5-(3) and (4). Hence Theorem 1 is shown.

\section{Proof of Theorem $3-5$}

In this section, we show that the measure of the subset $\mathcal{O}_{\varepsilon_{0}}$ is positive for the equations (3) - (5), which implies the growths of Sobolev norm.

\subsection{Proof of Theorem 3}

For Eq. (3),$E \in \mathbb{R}$, the corresponding linear system is

$$
\left(\begin{array}{l}
x \\
\xi
\end{array}\right)^{\prime}=\left[\left(\begin{array}{cc}
0 & E \\
-E & 0
\end{array}\right)+\left(\begin{array}{cc}
b(\omega t) & c(\omega t) \\
-a(\omega t) & -b(\omega t)
\end{array}\right)\right]\left(\begin{array}{c}
x \\
\xi
\end{array}\right) .
$$

In view of Lemma 5 of [14], for "generic" $a, b, c \in C^{\omega}\left(\mathbb{T}^{d}, \mathbb{R}\right)$, there is at least one non-degenerate $\tilde{\Lambda}_{k}, k \in \mathbb{Z}^{d}$. More precisely, at the resonant step of KAM scheme described in the proof of Proposition 5- (1), the condition (34) defines a resonant interval of $E$, on which the two eigenvalues $\pm \mathrm{i} \xi_{j}$ of $A_{j}$ are purely imaginary since $\xi_{j}$ is bounded frow below. After this resonant step, the two new eigenvalues $\pm \mathrm{i} \xi_{j+1}$ of $A_{j+1}$ can be real or still purely imaginary 
for $E$ in this resonant interval, since $\left|\xi_{j+1}\right|$ is close to zero. We say that $a, b, c \in C^{\omega}\left(\mathbb{T}^{d}, \mathbb{R}\right)$ are generic if, for at least one resonant step in the KAM scheme, the two new eigenvalues $\pm \mathrm{i} \xi_{j+1}$ become real on a sub-interval of the resonant interval.

\subsection{Proof of Theorem 4}

For Eq. (44) with $E \in \mathcal{I}=\left[E_{0}, E_{1}\right]$ with $E_{0}>0$ large enough, and $E_{1}<\infty$, Theorem 1 and 2 hold. The corresponding linear system $\left(\omega, A_{0}+F_{0}\right)$ of Eq. (41) is

$$
\left(\begin{array}{l}
x \\
\xi
\end{array}\right)^{\prime}=\left[\left(\begin{array}{cc}
0 & \sqrt{E} \\
-\sqrt{E} & 0
\end{array}\right)+\frac{q(\omega t)}{2 \sqrt{E}}\left(\begin{array}{cc}
-1 & -1 \\
1 & 1
\end{array}\right)\right]\left(\begin{array}{l}
x \\
\xi
\end{array}\right) .
$$

Then, through the change of variables

$$
\left(\begin{array}{l}
x \\
\xi
\end{array}\right)=\frac{1}{2 \sqrt{E}}\left(\begin{array}{cc}
\sqrt{E} & -1 \\
\sqrt{E} & 1
\end{array}\right)\left(\begin{array}{c}
\tilde{x} \\
\tilde{\xi}
\end{array}\right)
$$

$\left(\omega, A_{0}+F_{0}\right)$ is conjugated to

$$
\left(\begin{array}{c}
\tilde{x} \\
\tilde{\xi}
\end{array}\right)^{\prime}=C_{q}^{E}(\omega t)\left(\begin{array}{c}
\tilde{x} \\
\tilde{\xi}
\end{array}\right):=\left(\begin{array}{cc}
0 & 1 \\
-E+q(\omega t) & 0
\end{array}\right)\left(\begin{array}{c}
\tilde{x} \\
\tilde{\xi}
\end{array}\right) .
$$

The quasi-periodic linear system $\left(\omega, C_{q}^{E}(\cdot)\right)$ corresponds exactly to the eigenvalue problem of the quasi-periodic continuous Schrödinger operator $\mathcal{L}_{\omega, q}$ :

$$
\left(\mathcal{L}_{\omega, q} y\right)(t)=-y^{\prime \prime}(t)+q(\omega t) y(t) .
$$

By Gap labeling Theorem [28], if $\tilde{\Lambda}_{k}$ is not empty for $k \in \mathbb{Z}^{d}$, then it is indeed a "spectral gap" of $\mathcal{L}_{\omega, q}$ intersecting $\left[E_{0}, E_{1}\right]$, i.e., a connected component of $\left[E_{0}, E_{1}\right] \backslash \Sigma_{\omega, q}$ with $\Sigma_{\omega, q}$ denoting the spectrum of $\mathcal{L}_{\omega, q}$. In view of Theorem $\mathrm{C}$ of [14], for a generic potential $q$ (in the $|q|_{r}$-topology), for $E_{0}>0$ large enough, $\left[E_{0}, \infty\left[\cap \Sigma_{\omega, q}\right.\right.$ is a Cantor set. Hence there are infinitely many $\tilde{\Lambda}_{k}$ 's satisfying $\operatorname{Leb}\left(\tilde{\Lambda}_{k}\right)>0$.

\subsection{Proof of Theorem 5}

For Eq. (5) with $\nu(E)=\cos ^{-1}\left(-\frac{E}{2}\right), E \in[-2+\delta, 2-\delta]$ with $\delta>0$ a sufficiently small numerical constant (e.g. $\delta:=10^{-6}$ ), we can apply Theorem 1 and 2, if $a, b, c:[-2+\delta, 2-\delta] \times \mathbb{T}^{2} \rightarrow \operatorname{sl}(2, \mathbb{R})$ are small enough as assumed in Theorem 1 . 
For the quasi-periodic Schrödinger cocycle $\left(\alpha, S_{E}^{\lambda}\right)$

$$
X_{n+1}=S_{E}^{\lambda}(\theta+n \alpha) X_{n}=\left[\left(\begin{array}{cc}
-E & -1 \\
1 & 0
\end{array}\right)+\left(\begin{array}{cc}
2 \lambda \cos (\theta+n \alpha) & 0 \\
0 & 0
\end{array}\right)\right] X_{n},
$$

with $\alpha \in \mathrm{DC}_{1}(\gamma, \tau),|\lambda|$ small enough, it can be written as

$$
X_{n+1}=e^{B(E)} e^{G(E, \theta+n \alpha)} X_{n},
$$

for $e^{B(E)}:=\left(\begin{array}{cc}-E & -1 \\ 1 & 0\end{array}\right)$ and some $G(E, \cdot) \in \operatorname{sl}(2, \mathbb{R})$. This cocycle is related to the almost-Mathieu operator $H_{\lambda, \alpha, \theta}$ on $\ell^{2}(\mathbb{Z})$ :

$$
\left(H_{\lambda, \alpha, \theta} \psi\right)_{n}=-\left(\psi_{n+1}+\psi_{n-1}\right)+2 \lambda \cos (\theta+n \alpha) \psi_{n}, \quad n \in \mathbb{Z} .
$$

It is known that its spectrum, denoted by $\Sigma_{\lambda, \alpha}$, is a Cantor set [1], which is well-known as Ten Martini Problem. In fact, Avila-Jitomirskaya [2] further show that all spectral gaps are "open", which means that, for every $k \in \mathbb{Z}$,

$$
\tilde{\Lambda}_{k}:=\left\{E \in \mathbb{R}: \tilde{\rho}_{\left(\alpha, S_{E}^{\lambda}\right)}=\frac{k \alpha}{2} \bmod \mathbb{Z}\right\}
$$

has positive measure. Indeed, the size of $\tilde{\Lambda}_{k}$ decays exponentially with respect to $|k|$, as was shown in [30]. Here, $\tilde{\rho}_{\left(\alpha, S_{E}^{\lambda}\right)}$ is the fibered rotation number of cocycle $\left(\alpha, S_{E}^{\lambda}\right)$. Recall that for any $A: \mathbb{T}^{d} \rightarrow \operatorname{SL}(2, \mathbb{R})$ is continuous and homotopic to the identity, fibered rotation number of $(\alpha, A)$ is defined as

$$
\tilde{\rho}(\alpha, A)=\int \psi d \tilde{\mu} \bmod \mathbb{Z}
$$

where $\psi: \mathbb{T}^{d+1} \rightarrow \mathbb{R}$ is lift of $A$ such that

$$
A(x) \cdot(\cos 2 \pi y \sin 2 \pi y)=u(x, y)(\cos 2 \pi(y+\psi(x, y)) \sin 2 \pi(y+\psi(x, y)))
$$

and $\tilde{\mu}$ is invariant probability measure of $(x, y) \mapsto(x+\alpha, y+\psi(x, y))$ (according to [27], it does not depend on the choices of $\psi, \tilde{\mu})$.

Note that $\left(\alpha, S_{E}^{\lambda}\right)$ is a discrete dynamical system, however, with the help of Local Embedding Theorem (Theorem 8), we can embed the cocycle $\left(\alpha, S_{E}^{\lambda}\right)$ into a quasi-periodic linear system $(\omega, B(E)+F(E, \cdot))$. For an individual cocycle, the Local Embedding Theorem was already shown in 
[41]. Nevertheless, the crucial point here is that we really need a parameterized version of Local Embedding Theorem, that means the embedded system $(\omega, B(E)+F(E, \cdot))$ should have smooth dependence on $E$.

To show the parameterized version of Local Embedding Theorem, let us first introduce more notations. Given $f \in C^{2}(\mathcal{I})$, define

$$
|f|_{*}=\sum_{0 \leq m \leq 2} \sup _{E \in \mathcal{I}}\left|f^{(m)}\right|
$$

For any $f(E, \theta)=\sum_{k \in \mathbb{Z}^{d}} \widehat{f_{k}}(E) e^{2 \pi \mathrm{i}\langle k, \theta\rangle}$ which is $C^{2}$ w.r.t. $E \in \mathcal{I}, C^{\omega}$ w.r.t. $\theta \in \mathbb{T}^{d}$, denote

$$
\|f\|_{h}:=\sum_{k \in \mathbb{Z}^{d}}\left|\widehat{f}_{k}(E)\right|_{*} e^{2 \pi|k| h}<\infty
$$

and we denote by $C_{h}^{\omega}\left(\mathcal{I} \times \mathbb{T}^{d}, \mathbb{C}\right)$ all these functions with $\|f\|_{h}<\infty$. Then our result is the following:

Theorem 8. [Local Embedding Theorem] Given $d \geq 2, h>0$ and $G \in$ $C_{h}^{\omega}\left(\mathcal{I} \times \mathbb{T}^{d-1}, \operatorname{sl}(2, \mathbb{R})\right)$, suppose that $\mu \in \mathbb{T}^{d-1}$ such that $(1, \mu)$ is rationally independent. Then, for any $\nu \in C^{2}(\mathcal{I})$ satisfying

$$
\sup _{E \in \mathcal{I}}\left|\nu^{\prime}(E)\right| \cdot|\mathcal{I}|<\frac{1}{6}
$$

there exist $\epsilon=\epsilon\left(|\nu|_{*}, h,|\mu|\right)>0, c=c\left(|\nu|_{*}, h,|\mu|\right)>0$, and $F \in C_{\frac{h}{1+|\mu|}}^{\omega}(\mathcal{I} \times$ $\left.\mathbb{T}^{d}, \operatorname{sl}(2, \mathbb{R})\right)$ such that the cocycle $\left(\mu, e^{2 \pi \nu J} e^{G(\cdot)}\right)$ is the Poincaré map of linear system

$$
\left(\begin{array}{l}
x \\
\xi
\end{array}\right)^{\prime}=(\nu J+F(\omega t))\left(\begin{array}{l}
x \\
\xi
\end{array}\right), \quad \omega=(1, \mu)
$$

provided that $\|G\|_{h}<\epsilon$. Moreover, we have $\|F\|_{\frac{h}{1+|\mu|}} \leq 2 c\|G\|_{h}$.

We postpone the proof of Theorem 8 to Appendix Appendix A.

Now let us show how we can apply Theorem 8 to finish the proof of Theorem 5. First note the constant matrix $e^{B}$ can be rewritten as

$$
e^{B}:=\left(\begin{array}{cc}
-E & -1 \\
1 & 0
\end{array}\right)=M\left(\begin{array}{cc}
\cos (\nu) & -\sin (\nu) \\
\sin (\nu) & \cos (\nu)
\end{array}\right) M^{-1},
$$


where

$$
M:=\frac{1}{\sqrt{\sin (\nu)}}\left(\begin{array}{cc}
\cos (\nu) & -\sin (\nu) \\
1 & 0
\end{array}\right)
$$

recalling that

$$
\cos (\nu(E))=-\frac{E}{2}, \quad \sin (\nu(E))=\frac{\sqrt{4-E^{2}}}{2}, \quad E \in[-2+\delta, 2-\delta] .
$$

Hence, by noting

$$
\left(\begin{array}{cc}
\cos (\nu) & -\sin (\nu) \\
\sin (\nu) & \cos (\nu)
\end{array}\right)=\exp \left\{\left(\begin{array}{cc}
0 & -\nu \\
\nu & 0
\end{array}\right)\right\}
$$

we see that $B$ can be written as $B=M \cdot(\nu J) \cdot M^{-1}$.

For $\nu(E)=\cos ^{-1}\left(-\frac{E}{2}\right)$, there exists $\mathcal{I} \subset[-2+\delta, 2-\delta]$ such that (37) is satisfied. For example, we can take $\mathcal{I}=]-\frac{2}{\sqrt{37}}, \frac{2}{\sqrt{37}}[$. Therefore, according to Theorem 8 , for $\omega \in(1, \alpha)$, we have a quasi-periodic linear system $(\omega, B(E)+$ $F(E, \cdot))$ from the quasi-periodic Schrödinger cocycle $\left(\alpha, S_{E}^{\lambda}\right)$ :

$$
\left(\begin{array}{l}
x \\
\xi
\end{array}\right)^{\prime}=(B(E)+F(E, \omega t))\left(\begin{array}{l}
x \\
\xi
\end{array}\right)
$$

Through the change of variables

$$
\left(\begin{array}{l}
x \\
\xi
\end{array}\right)=M\left(\begin{array}{c}
\tilde{x} \\
\tilde{\xi}
\end{array}\right)
$$

$(\omega, B(E)+F(E, \cdot))$ is conjugated to

$$
\left(\begin{array}{c}
\tilde{x} \\
\tilde{\xi}
\end{array}\right)^{\prime}=\left(\left(\begin{array}{cc}
0 & -\nu \\
\nu & 0
\end{array}\right)+M F(E, \omega t) M^{-1}\right)\left(\begin{array}{c}
\tilde{x} \\
\tilde{\xi}
\end{array}\right) .
$$

Then by Theorem 1 and 2 , Theorem 5 is shown with

$$
\left(\begin{array}{cc}
b(E, \cdot) & c(E, \cdot) \\
-a(E, \cdot) & -b(E, \cdot)
\end{array}\right)=M F(E, \cdot) M^{-1} .
$$

Finally we point out that $\rho_{(\omega, B(E)+F(E, \cdot))}=\tilde{\rho}_{\left(\alpha, S_{E}^{\lambda}(\cdot)\right)}$, since $\left(\alpha, S_{E}^{\lambda}\right)$ is the Poincaré map of linear system $(\omega, B(E)+F(E, \cdot))$. Let

$$
\tilde{\Lambda}_{(-p, k)}:=\left\{E \in \overline{\mathcal{I}}: \rho_{(\omega, B(E)+F(E, \cdot))}=\frac{k \alpha-p}{2}=\min _{j \in \mathbb{Z}}\left|\frac{k \alpha}{2}-j\right|\right\},
$$

then by well-known result of Avila-Jitomirskaya [2], $\operatorname{Leb}\left(\tilde{\Lambda}_{(-p, k)}\right)>0$, for every $k \in \mathbb{Z}$ such that $\tilde{\Lambda}_{k}$ intersect with $\mathcal{I}$. 


\section{Appendix A. Proof of Theorem 8}

The main ideas of the proof will follow Theorem 3.2 of [41], we sketch the proof and point out the differences. First we need the following key observations.

Lemma Appendix A.1. For any $k \in \mathbb{Z}^{d-1}$, and for any $\nu \in C^{2}(\mathcal{I})$ satisfying (37), there exists $\tilde{k}=\tilde{k}(k) \in \mathbb{Z}$ which is independent of $E$, such that

$$
|\langle k, \mu\rangle+2 \nu+\tilde{k}| \in\left[0, \frac{5}{6}\right], \quad \forall E \in \mathcal{I} .
$$

Proof: For any given $E$, we can define $\tilde{k}=\tilde{k}(k, E) \in \mathbb{Z}$ by

$$
|\langle k, \mu\rangle+2 \nu(E)+\tilde{k}|=\inf _{j \in \mathbb{Z}}|\langle k, \mu\rangle+2 \nu(E)+j|,
$$

we only need to show that $\tilde{k}$ can be chosen independent of $E$.

To do this, we only need to consider two extreme cases. If there exists $E_{0} \in \mathcal{I}$ such that $\inf _{k \in \mathbb{Z}}\left|\langle k, \mu\rangle+2 \nu\left(E_{0}\right)+k\right|=0$, then $\tilde{k}(k)$ is uniquely defined, and by assumption (37),

$$
|\langle k, \mu\rangle+2 \nu(E)+\tilde{k}| \leq 2\left|\nu(E)-\nu\left(E_{0}\right)\right| \leq 2 \sup _{E \in \mathcal{I}}\left|\nu^{\prime}(E)\right| \cdot|\mathcal{I}|<\frac{1}{3} .
$$

If there exists $E_{0} \in \mathcal{I}$ such that $\inf _{k \in \mathbb{Z}}\left|\langle k, \mu\rangle+2 \nu\left(E_{0}\right)+k\right|=\frac{1}{2}$, then $\tilde{k}(k)$ is not uniquely defined, and one can choose $\tilde{k}(k)$ to be the smaller one which satisfies (A.1). By assumption (37), one has

$$
\begin{aligned}
|\langle k, \mu\rangle+2 \nu(E)+\tilde{k}| & \leq\left|\langle k, \mu\rangle+2 \nu\left(E_{0}\right)+\tilde{k}\right|+2\left|\nu(E)-\nu\left(E_{0}\right)\right| \\
& \leq \frac{1}{2}+2 \sup _{E \in \mathcal{I}}\left|\nu^{\prime}(E)\right| \cdot|\mathcal{I}| \\
& <\frac{5}{6} .
\end{aligned}
$$

Once we have Lemma Appendix A.1, we can define the resonance sites $\mathcal{S} \subset \mathbb{Z}^{d}$ as follows

$$
\mathcal{S}:=\left\{(\tilde{k}, k): k \in \mathbb{Z}^{d-1}\right\}
$$


For any $f\left(E, \theta_{1}, \tilde{\theta}\right)=\sum_{k \in \mathbb{Z}^{d-1}} \widehat{f}_{\tilde{k}, k}(E) e^{2 \pi \mathrm{i}\left(\tilde{k} \theta_{1}+\langle k, \tilde{\theta}\rangle\right)} \in C_{h}^{\omega}\left(\mathcal{I} \times \mathbb{T}^{d}, \mathbb{C}\right)$, we define its weighted norm by

$$
\|f\|_{\nu, h}^{\mu}:=\sum_{k \in \mathbb{Z}^{d-1}}\left|\widehat{f}_{\tilde{k}, k}(E)\right|_{*} e^{2 \pi|k|(1+|\mu|) h},
$$

and then we can define the linear sub-space $\mathcal{B}_{\nu, h}^{\mu}\left(\mathcal{I} \times \mathbb{T}^{d}, \mathbb{C}\right)$ of $C_{h}^{\omega}\left(\mathcal{I} \times \mathbb{T}^{d}, \mathbb{C}\right)$

$\mathcal{B}_{\nu, h}^{\mu}\left(\mathcal{I} \times \mathbb{T}^{d}, \mathbb{C}\right):=\left\{f: f\left(E, \theta_{1}, \tilde{\theta}\right)=\sum_{k \in \mathbb{Z}^{d-1}} \widehat{f}_{\tilde{k}, k}(E) e^{2 \pi \mathrm{i}\left(\tilde{k} \theta_{1}+\langle k, \tilde{\theta}\rangle\right)},\|f\|_{\nu, h}^{\mu}<\infty\right\}$

In the following, we will show that $\mathcal{B}_{\nu, \frac{h}{1+|\mu|}}^{\mu}\left(\mathcal{I} \times \mathbb{T}^{d}, \mathbb{C}\right)$ is actually isomorphic to $C_{h}^{\omega}\left(\mathcal{I} \times \mathbb{T}^{d-1}, \mathbb{C}\right)$, therefore a Banach space. The space will be used to construct the embedded linear system.

Proposition 6. For any $\nu \in C^{2}(\mathcal{I})$ satisfying (37), the linear operator

$$
\begin{aligned}
T: \mathcal{B}_{\nu, \frac{h}{1+|\mu|}}^{\mu}\left(\mathcal{I} \times \mathbb{T}^{d}, \mathbb{C}\right) & \rightarrow C_{h}^{\omega}\left(\mathcal{I} \times \mathbb{T}^{d-1}, \mathbb{C}\right) \\
f(E, \theta) & \mapsto \int_{0}^{1} f(E, t, \tilde{\theta}+t \mu) e^{4 \pi \mathrm{i} \nu(E) t} d t
\end{aligned}
$$

is bounded. Moreover, there exists numerical constant $c>0$ such that

$$
T^{-1}: C_{h}^{\omega}\left(\mathcal{I} \times \mathbb{T}^{d-1}, \mathbb{C}\right) \rightarrow \mathcal{B}_{\nu, \frac{h}{1+|\mu|}}^{\mu}\left(\mathcal{I} \times \mathbb{T}^{d}, \mathbb{C}\right)
$$

is also bounded with estimate $\left\|T^{-1}\right\| \leq c|\nu|_{*}$.

Before giving the proof of Proposition 6 , we introduce the following auxiliary function, which is quite important for the proof.

Lemma Appendix A.2. For the function

$$
H(x)=\left\{\begin{array}{cc}
\frac{e^{2 \pi \mathrm{i} x}-1}{2 \pi \mathrm{i} x}, & x \neq 0 \\
1, & x=0
\end{array}\right.
$$

we have $H, \frac{1}{H} \in C^{\infty}\left[-\frac{5}{6}, \frac{5}{6}\right]$ and

$$
|H(x)| \in\left[\frac{3}{5 \pi}, 1\right] \quad \forall|x| \leq \frac{5}{6} .
$$



Since

Proof: By Taylor expansions, one can easily check that $H \in C^{\infty}\left[-\frac{5}{6}, \frac{5}{6}\right]$.

$$
|H(x)|=\left|\frac{\sin (\pi x)}{\pi x}\right|,
$$

then (A.2) follows from the simple fact

$$
\frac{2}{\pi}|t|<|\sin (t)| \leq|t|, \quad t \in\left[0, \frac{\pi}{2}\right] .
$$

Consequently, $H^{-1}$ is also a $C^{\infty}$ function.

Proof of Proposition 6. For any $f \in \mathcal{B}_{\nu, \frac{h}{1+|\mu|}}^{\mu}\left(\mathcal{I} \times \mathbb{T}^{d}, \mathbb{C}\right)$, direct computations show that

$$
T f(E, \theta)=\sum_{k \in \mathbb{Z}^{d-1}} \sum_{(\tilde{k}, k) \in \mathcal{S}} \widehat{f}_{\tilde{k}, k}(E) H(\langle k, \mu\rangle+2 \nu(E)+\tilde{k}) e^{2 \pi \mathrm{i}\langle k, \theta\rangle} .
$$

Here we shall use the crucial fact that $\tilde{k}$ is independent of $E$, thus by Lemma Appendix A.1, $\langle k, \mu\rangle+2 \nu(E)+\tilde{k} \in C^{2}(\mathcal{I})$, and $|\langle k, \mu\rangle+2 \nu(E)+\tilde{k}| \leq$ $\frac{5}{6}$. By Lemma Appendix A.2, $H(\langle k, \mu\rangle+2 \nu(E)+\tilde{k})$ is well defined and $H(\langle k, \mu\rangle+2 \nu(E)+\tilde{k}) \in C^{2}(\mathcal{I})$. Consequently, there exists numerical constant $c$ such that

$$
\|T f\|_{h}=\sum_{k \in \mathbb{Z}^{d-1}}\left|(\widehat{T f})_{k}\right|_{*} e^{2 \pi|k| h} \leq c|\nu|_{*}\|f\|_{\nu, \frac{h}{1+|\mu|}}^{\mu}
$$

Hence $T$ is a bounded linear operator.

On the other hand, for any $\varphi \in C_{h}^{\omega}\left(\mathcal{I} \times \mathbb{T}^{d-1}, \mathbb{C}\right)$, we write

$$
\varphi(E, \theta)=\sum_{k \in \mathbb{Z}^{d-1}} \widehat{\varphi}_{k}(E) e^{2 \pi \mathrm{i}\langle k, \theta\rangle} .
$$

Define $\widehat{f}_{k_{1}, k}(E)$ by

$$
\widehat{f}_{k_{1}, k}(E)=\left\{\begin{array}{cc}
\frac{\hat{\varphi}_{k}(E)}{H(\langle k, \mu\rangle+2 \nu(E)+\tilde{k})}, & k_{1}=\tilde{k} \\
0, & k_{1} \neq \tilde{k}
\end{array}\right.
$$

where $(\tilde{k}, k) \in \mathcal{S}$. Then one can check that

$$
f\left(E, \theta_{1}, \tilde{\theta}\right)=\sum_{k \in \mathbb{Z}^{d-1}} \widehat{f}_{k_{1}, k}(E) e^{2 \pi \mathrm{i}\left(k_{1} \theta_{1}+\langle k, \tilde{\theta}\rangle\right)} \in \mathcal{B}_{\nu, h}^{\mu}\left(\mathcal{I} \times \mathbb{T}^{d}, \mathbb{C}\right)
$$


is uniquely defined and it satisfies $T f(E, 0, \tilde{\theta})=\varphi(E, \tilde{\theta})$. By Lemma Appendix A.1 and Lemma Appendix A.2, $H^{-1}(\langle k, \mu\rangle+2 \nu(E)+\tilde{k}) \in C^{2}(\mathcal{I})$, consequently, we have

$$
\|f\|_{\nu, \frac{h}{1+|\mu|}}^{\mu} \leq c|\nu|_{*}\|\varphi\|_{h}
$$

Hence $\left\|T^{-1}\right\| \leq c|\nu|_{*}$.

For any $\nu \in C^{2}(\mathcal{I})$, we then can define the Banach space

$$
\overline{\mathcal{B}}=\left\{\left(\begin{array}{cc}
\mathrm{i} f & g \\
\bar{g} & -\mathrm{i} f
\end{array}\right): f \in \mathcal{B}_{0, \frac{h}{1+|\mu|}}^{\mu}\left(\mathcal{I} \times \mathbb{T}^{d}, \mathbb{R}\right), g \in \mathcal{B}_{-\nu, \frac{h}{1+|\mu|}}^{\mu}\left(\mathcal{I} \times \mathbb{T}^{d}, \mathbb{C}\right)\right\}
$$

then $\overline{\mathcal{B}} \subset C_{\frac{h}{1+|\mu|}}^{\omega}\left(\mathcal{I} \times \mathbb{T}^{d}, \operatorname{su}(1,1)\right)$. Note the algebra $\operatorname{su}(1,1)$ and $\operatorname{sl}(2, \mathbb{R})$ are isomorphic with isomorphism given by $B \rightarrow \bar{M}^{-1} B \bar{M}$ where

$$
\bar{M}=\left(\begin{array}{cc}
1 & -\mathrm{i} \\
1 & \mathrm{i}
\end{array}\right)
$$

Therefore, we have $\mathcal{B}:=\bar{M}^{-1} \overline{\mathcal{B}} \bar{M} \subset C_{\frac{h}{1+|\mu|}}^{\omega}\left(\mathcal{I} \times \mathbb{T}^{d}, \operatorname{sl}(2, \mathbb{R})\right)$.

As a corollary of Proposition 6, we have the following:

Corollary 1. For any $\nu \in C^{2}(\mathcal{I})$ satisfying (37), then the linear operator

$$
\begin{aligned}
L: \mathcal{B} & \rightarrow C_{h}^{\omega}\left(\mathcal{I} \times \mathbb{T}^{d-1}, \operatorname{sl}(2, \mathbb{R})\right) \\
F & \mapsto \int_{0}^{1} e^{-2 \pi \nu J s} F(s, \theta+s \mu) e^{2 \pi \nu J s} d s
\end{aligned}
$$

is bounded. Moreover, there exists numerical constant $c>0$ such that

$$
L^{-1}: C_{h}^{\omega}\left(\mathcal{I} \times \mathbb{T}^{d-1}, \operatorname{sl}(2, \mathbb{R})\right) \rightarrow \mathcal{B}
$$

is bounded with $\left\|L^{-1}\right\| \leq c|\nu|_{*}$.

Proof: It is an immediate corollary of corollary of Proposition 6, similar proof can be found in Corollary 3.1 of [41]. We omit the details.

Proof of Theorem 8 . Now we can finish the whole proof of Theorem 8 . We will use quantitative Implicit Function Theorem (c.f. Theorem 3.1 of [41]) to prove the result. Suppose that $\Phi^{t}(E, \theta)$ is the fundamental solution matrix of (38),

$$
\Phi^{t}(E, \theta)=e^{2 \pi \nu(E) J t}\left(\mathrm{Id}+\int_{0}^{t} e^{-2 \pi \nu(E) J s} F(E, \theta+s \omega) \Phi^{s}(E, \theta) d s\right),
$$


where Id denotes the identity matrix.

We will show that the cocycle $\left(\mu, e^{2 \pi \nu J} e^{G(E, \tilde{\theta})}\right)$ can be embedded into the linear system ( $(38)$, which means $\Phi^{1}(E, 0, \tilde{\theta})=e^{2 \pi \nu(E) J} e^{G(E, \tilde{\theta})}$, i.e.,

$$
e^{2 \pi \nu J}\left(\operatorname{Id}+\int_{0}^{1} e^{-2 \pi \nu J s} F(E, s, \tilde{\theta}+s \mu) \Phi^{s}(E, 0, \tilde{\theta}) d s\right)=e^{2 \pi \nu J} e^{G(E, \tilde{\theta})} .
$$

We then construct the nonlinear functional

$$
\Psi: \mathcal{B} \times C_{h}^{\omega}\left(\mathcal{I} \times \mathbb{T}^{d-1}, \operatorname{sl}(2, \mathbb{R})\right) \rightarrow C_{h}^{\omega}\left(\mathcal{I} \times \mathbb{T}^{d-1}, \operatorname{gl}(2, \mathbb{R})\right)
$$

by defining

$$
\Psi(F, G):=\mathrm{Id}+\int_{0}^{1} e^{-2 \pi \nu J s} F(E, s, \tilde{\theta}+s \mu) \Phi^{s}(E, 0, \tilde{\theta}) d s-e^{G(E, \tilde{\theta})}
$$

Immediate check shows that $\Psi(0,0)=0,\|\Psi(0, G)\| \leq\|G\|_{h}$, and

$$
\begin{aligned}
D_{F} \Psi(F, G)(\widetilde{F})= & \int_{0}^{1} e^{-2 \pi \nu J s} \widetilde{F}(E, s, \tilde{\theta}+s \omega) \Phi^{s}(E, 0, \tilde{\theta}) d s \\
& +\int_{0}^{1} e^{-2 \pi \nu J s} F(E, s, \tilde{\theta}+s \mu) D_{F} \Phi^{s}(0, \tilde{\theta}) \widetilde{F}(E, s, \tilde{\theta}+s \mu) d s .
\end{aligned}
$$

Consequently, we have

$$
D_{F} \Psi(0,0)(\widetilde{F})=\int_{0}^{1} e^{-2 \pi \nu J s} \widetilde{F}(E, s, \tilde{\theta}+s \mu) e^{2 \pi \nu J s} d s .
$$

By Corollary 1, $D_{F} \Psi(0,0)^{-1}: C_{h}^{\omega}\left(\mathcal{I} \times \mathbb{T}^{d-1}, \operatorname{sl}(2, \mathbb{R})\right) \rightarrow \mathcal{B}$ is a bounded linear operator with estimate $\left\|D_{F} \Psi(0,0)^{-1}\right\| \leq c|\nu|_{*}$.

The rest proof are quite standard, one can consult Theorem 3.2 of [41] for details, we omit the details.

\section{Acknowledgements}

The authors would like to thank Prof. D. Bambusi and Prof. J. You for their interests and fruitful discussions which helped to improve the proof. They also appreciate the anonymous referees for helpful remarks and suggestions in modifying this manuscript. Z. Zhao would like to thank the support of Visiting Scholars of Shanghai Jiaotong University (SJTU) and Visiting Scholars of Chern Institute of Mathematics (CIM) during his visits.

\section{References}




\section{References}

[1] Avila, A., Jitomirskaya, S.: The Ten Martini Problem. Ann. of Math., 170, 303-342 (2009).

[2] Avila, A., Jitomirskaya, S.: Almost localization and almost reducibility. J. Eur. Math. Soc., 12, 93-131 (2010).

[3] Bambusi, D.: Reducibility of 1-d Schrödinger equation with time quasiperiodic unbounded perturbations. I. Trans. Amer. Math. Soc., 370, 1823-1865, (2018).

[4] Bambusi, D.: Reducibility of 1-d Schrödinger equation with time quasiperiodic unbounded perturbations. II. Commun. Math. Phys., 353(1), 353-378, (2017).

[5] Bambusi, D., Langella, D., Montalto, R.: Reducibility of non-resonant transport equation on with unbounded perturbations. Ann. Henri Poincaré, 20, 1893-1929 (2019).

[6] Bambusi, D., Graffi, S.: Time quasi-periodic unbounded perturbations of Schrödinger operators and KAM methods. Commun. Math. Phys.,

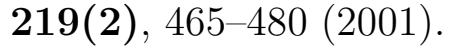

[7] Bambusi, D., Grébert, B., Maspero, A., Robert, D.: Reducibility of the quantum harmonic oscillator in $d$-dimensions with polynomial time dependent perturbation. Analysis and PDEs, 11(3), 775-799 (2018).

[8] Bambusi, D., Grébert, B., Maspero, A., Robert, D.: Growth of Sobolev norms for abstract linear Schrödinger equations. To appear in J. Eur. Math. Soc. (JEMS), online 2020.

[9] Bambusi, D., Montalto, R.: Reducibility of 1-d Schrödinger equation with unbounded time quasiperiodic perturbations. III. J. Math. Phys., 59, 122702 (2018).

[10] Bourgain, J.: Growth of Sobolev norms in linear Schrödinger equations with quasi-periodic potential. Commun. Math. Phys., 204(1), 207-247 (1999). 
[11] Combescure, M.: The quantum stability problem for time-periodic perturbations of the harmonic oscillator. Ann. Inst. H. Poincaré Phys. Théor., 47(1), 63-83 (1987).

[12] Delort, J.-M.: Growth of Sobolev norms for solutions of time dependent Schrödinger operators with harmonic oscillator potential. Comm. Partial Differential Equations, 39(1), 1-33 (2014).

[13] Duclos, P., Lev, O., Št'ovíček, P., Vittot, M.: Weakly regular Floquet Hamiltonians with pure point spectrum. Rev. Math. Phys. 14(6), 531568 (2002).

[14] Eliasson, L. H.: Floquet solutions for the 1-dimensional quasi-periodic Schrödinger equation. Commun. Math. Phys., 146, 447-482 (1992).

[15] Eliasson, L. H., Kuksin S. B.: On reducibility of Schrödinger equations with quasiperiodic in time potentials. Commun. Math. Phys., 286(1), 125-135 (2009).

[16] Eliasson, L. H., Kuksin S. B.: KAM for the nonlinear Schrödinger equation. Ann. of Math. 172(1), 371-435 (2010).

[17] Enss, V., Veselic, K.: Bound states and propagating states for timedependent hamiltonians. Ann IHP, 39(2), 159-191 (1983).

[18] Faou, E., Raphaël, P.: On weakly turbulent solutions to the perturbed linear harmonic oscillator. arXiv:2006.08206.

[19] Feola, R., Giuliani, F., Montalto, R., Procesi, M.: Reducibility of first order linear operators on tori via Moser's theorem. J. Funct. Anal., 276(3), 932-970 (2019).

[20] Feola, R., Grébert, B.: Reducibility of Schrödinger equation on the sphere. arXiv:1905.11964.

[21] Feola, R., Grébert, B., Nguyen, T.: Reducibility of Schrödinger equation on a Zoll manifold with unbounded potential. arXiv:1910.10657.

[22] Graffi, S., Yajima, K.: Absolute continuity of the Floquet spectrum for a nonlinearly forced harmonic oscillator. Commun. Math. Phys., 215(2), 245-250 (2000). 
[23] Grébert, B., Thomann, L.: KAM for the quantum harmonic oscillator. Commun. Math. Phys., 307, 383-427 (2011).

[24] Grébert, B., Paturel, E.: On reducibility of quantum harmonic oscillator on $\mathbb{R}^{d}$ with quasi-periodic in time potential. Annales de la Faculté des sciences de Toulouse : Mathématiques, Série 6, Tome 28(5), 977-1014 (2019).

[25] Hadj Amor, S.: Hölder continuity of the rotation number for quasiperiodic co-cycles in $\mathrm{SL}(2, \mathbb{R})$. Commun. Math. Phys., 287(2), 565-588 (2009).

[26] Hagedorn, G., Loss, M., Slawny, J.: Nonstochasticity of time-dependent quadratic Hamiltonians and the spectra of canonical transformations. J. Phys. A, 19(4), 521- 531 (1986).

[27] Herman, M.: Une méthode pour minorer les exposants de Lyapounov et quelques exemples montrant le caractère local d'un théorème d'Arnold et de Moser sur le tore de dimension 2. Comment. Math. Helv., 58(3), 453-502 (1983).

[28] Johnson, R., Moser, J.: The rotation number for almost periodic potentials. Commun. Math. Phys., 84(3), 403-438 (1982).

[29] Kuksin, S., B.: Nearly integrable infinite-dimensional Hamiltonian systems. Lecture Notes in Mathematics 1556, Springer, 1993.

[30] Leguil, M., You, J., Zhao, Z., Zhou. Q.: Asymptotics of spectral gaps of quasi-periodic Schrödinger operators. arXiv:1712.04700.

[31] Liang, Z., Luo, J.: Reducibility of 1-d quantum harmonic oscillator equation with unbounded oscillation perturbations. To appear in J. Diff. Eqs.

[32] Liang, Z., Wang, Z.: Reducibility of quantum harmonic oscillator on $\mathbb{R}^{d}$ with differential and quasi-periodic in time potential. J. Diff. Eqs., 267, 3355-3395 (2019).

[33] Liu, J., Yuan, X.: Spectrum for quantum Duffing oscillator and smalldivisor equation with large-variable coefficient. Comm. Pure Appl. Math., 63(9), 1145-1172 (2010). 
[34] Maspero, A., Robert, D.: On time dependent Schrödinger equations: Global well-posedness and growth of Sobolev norms. J. Func. Anal., 273(2), 721-781 (2017).

[35] Maspero, A.: Lower bounds on the growth of Sobolev norms in some linear time dependent Schrödinger equations. Math. Res. Lett., 26(4), 1197-1215 (2019).

[36] Montalto, R.: A reducibility result for a class of linear wave equations on $\mathbb{T}^{d}$. Int. Math. Res. Notices, 2019(6), 1788-1862 (2019).

[37] Schwinte, V., Thomann, L.: Growth of Sobolev norms for coupled Lowest Landau Level equations. arXiv:2006.01468.

[38] Thomann, L.: Growth of Sobolev norms for linear Schrödinger operators. arXiv:2006.02674.

[39] Wang, W.-M.: Pure point spectrum of the Floquet Hamiltonian for the quantum harmonic oscillator under time quasi-periodic perturbations. Commun. Math. Phys., 277(2), 459-496 (2008).

[40] Wang, Z., Liang, Z.: Reducibility of 1d quantum harmonic oscillator perturbed by a quasiperiodic potential with logarithmic decay. Nonlinearity, 30(4), 1405-1448 (2017).

[41] You, J., Zhou, Q.: Embedding of analytic quasi-Periodic cocycles into analytic quasi-periodic linear systems and its applications. Commun. Math. Phys., 323(3), 975-1005 (2013). 